\title{
Interplay between Surface Chemistry, Precursor Reactivity, and Temperature Determines Outcome of ZnS Shelling Reactions on CulnS 2 Nanocrystals
}

Anne C. Berends, ${ }^{\dagger}$ Ward van der Stam, ${ }^{\dagger, \Phi \odot ~ J a n ~ P . ~ H o f m a n n, ~}{ }^{\ddagger}{ }^{\dagger}$ Eva Bladt, ${ }^{\S}$ Johannes D. Meeldijk, $^{\perp}$ Sara Bals, ${ }^{\S}$ and Celso de Mello Donega*, ${ }^{\dagger}$ (i)

${ }^{\dagger}$ Condensed Matter and Interfaces, Debye Institute for Nanomaterials Science, Utrecht University, Post Office Box 80000, 3508 TA Utrecht, The Netherlands

${ }^{\ddagger}$ Laboratory of Inorganic Materials Chemistry, Department of Chemical Engineering and Chemistry, Eindhoven University of Technology, Postbox 513, 5600 MB Eindhoven, The Netherlands

${ }^{\S}$ EMAT, Department of Physics, University of Antwerpen, Groenenborgerlaan 171, 2010 Antwerpen, Belgium

${ }^{\perp}$ Electron Microscopy Utrecht, Debye Institute for Nanomaterials Science, Utrecht University, $3584 \mathrm{CH}$ Utrecht, Netherlands

Supporting Information

\begin{abstract}
ZnS shelling of $\mathrm{I}-\mathrm{III}-\mathrm{VI}_{2}$ nanocrystals (NCs) invariably leads to blue-shifts in both the absorption and photoluminescence spectra. These observations imply that the outcome of $\mathrm{ZnS}$ shelling reactions on $\mathrm{I}-\mathrm{III}-\mathrm{VI}_{2}$ colloidal NCs results from a complex interplay between several processes taking place in solution, at the surface of, and within the seed NC. However, a fundamental understanding of the factors determining the balance between these different processes is still lacking. In this work, we address this need by investigating the impact of precursor reactivity, reaction temperature, and surface chemistry (due to the washing procedure) on the outcome of $\mathrm{ZnS}$ shelling reactions on $\mathrm{CuInS}_{2}$ NCs using a seeded growth approach. We demonstrate that low reaction

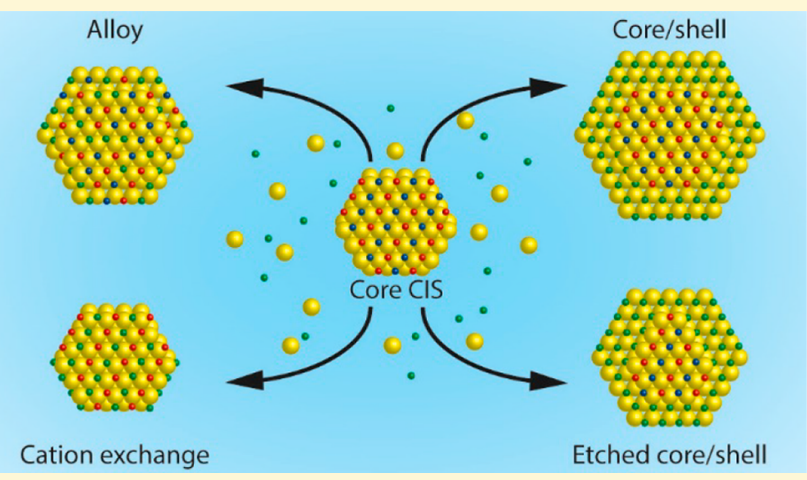
temperatures $\left(150^{\circ} \mathrm{C}\right)$ favor etching, cation exchange, and alloying regardless of the precursors used. Heteroepitaxial shell overgrowth becomes the dominant process only if reactive $\mathrm{S}$ - and $\mathrm{Zn}$-precursors ( $\mathrm{S}$-ODE/OLAM and $\mathrm{ZnI}_{2}$ ) and high reaction temperatures $\left(210^{\circ} \mathrm{C}\right)$ are used, although a certain degree of heterointerfacial alloying still occurs. Remarkably, the presence of residual acetate at the surface of CIS seed NCs washed with ethanol is shown to facilitate heteroepitaxial shell overgrowth, yielding for the first time CIS/ZnS core/shell NCs displaying red-shifted absorption spectra, in agreement with the spectral shifts expected for a type-I band alignment. The insights provided by this work pave the way toward the design of improved synthesis strategies to CIS/ZnS core/shell and alloy NCs with tailored elemental distribution profiles, allowing precise tuning of the optoelectronic properties of the resulting materials.
\end{abstract}

$\mathrm{T}$ he search for $\mathrm{Pb}$ - and Cd-free nanocrystals (NCs) has greatly intensified in recent years. Copper indium sulfide (CIS) in particular is one of the most extensively investigated alternative materials since CIS NCs display tunable photoluminescence (PL) through the visible and near-infrared (NIR) spectral window, large absorption cross-sections across a broad spectral range, and low toxicity. ${ }^{1-6}$ These properties make colloidal CIS NCs promising materials for a variety of applications (viz., light-emitting diodes, photovoltaics, bioimaging, and spectral converters in displays and luminescent solar concentrators). ${ }^{1-10}$ However, the PL quantum yield (QY) of CIS NCs is typically low $(<10 \%)^{1,3-5,11-20}$ due to exciton trapping at surface defects, which facilitates nonradiative decay. ${ }^{1,3-5,11-16,18,21-25}$ To realize the full potential of CIS NCs, it is thus imperative that strategies to boost their PL QYs are developed. The extensive research carried out over the last decades on the prototypical II-VI semiconductor NCs has clearly established that exciton trapping at surface defects can be effectively prevented by overgrowth of shells of wider band gap materials, thereby leading in recent years to CdSe-based core/shell quantum dots (QDs) with near unity ensemble PL QYs. $^{26-31}$ The excellent quality of II-VI core/shell QDs motivated the scientific community to develop similar strategies for CIS NCs. To date, these efforts have mainly focused on the overgrowth of $\mathrm{ZnS}$ shells since $\mathrm{ZnS}$ is a nontoxic, stable, and abundant material, with a small lattice mismatch with respect to CIS (viz., 2\%), and a wide band gap $(3.54 \mathrm{eV})$ with (bulk) band

Received: February 1, 2018

Revised: $\quad$ March 23, 2018

Published: March 25, 2018 
edges positioned such that a type-I band alignment with CIS is expected. ${ }^{6,13,19,21}$

Many studies on the preparation of CIS/ZnS core/shell NCs have indeed reported enhanced PL QYs (as high as 80\%) and increased stability. ${ }^{2,11-25,32-35}$ However, spectral blue-shifts in both absorption and PL have been invariably observed after $\mathrm{ZnS}$ shelling of CIS NCs, in striking contrast with the small red-shift expected for type-I core/shell hetero-NCs due to leakage of the exciton wave function into the shell. ${ }^{29}$ This widening of the band gap of the NCs after ZnS shelling has been attributed to a variety of reasons: formation of graded alloy (CIS,ZnS) NCs, ${ }^{15,16,25,35}$ etching of the CIS NC cores prior to onset of heteroepitaxial shell overgrowth, ${ }^{11,18,19}$ or superseded $\mathrm{ZnS}$ shell ingrowth by $\mathrm{Zn}^{2+}$ for $\mathrm{Cu}^{+}$and $\mathrm{In}^{3+}$ cation exchange. ${ }^{14,19}$ This implies that the outcome of $\mathrm{ZnS}$ shelling reactions on CIS NCs is dictated by a complex interplay between a number of processes, as schematically illustrated in Figure 1. Although this is to a certain extent true for any

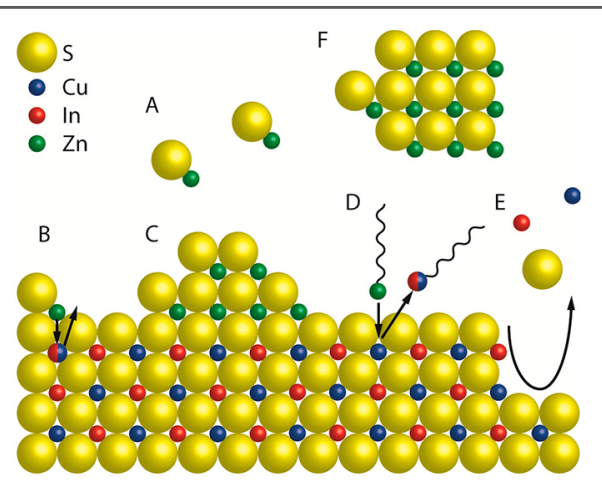

Figure 1. Schematic representation of a CIS NC surface depicting the chemical processes that can take place during a $\mathrm{ZnS}$ shelling reaction. (A) $[\mathrm{ZnS}]$ monomers form homogeneously in solution from $\mathrm{Zn}$ - and S-precursors. (B) Alloying: $\mathrm{Zn}^{2+}$ from adsorbed $[\mathrm{ZnS}]$ monomer units diffuses inward, while $\mathrm{Cu}^{+}$and/or $\mathrm{In}^{3+}$ ions diffuse outward. (C) Heteroepitaxial shell overgrowth. A stable $\mathrm{ZnS}$ phase grows on the CIS surface. (D) Cation exchange: upon adsorption of $\mathrm{Zn}-\mathrm{R}$ species at the CIS surface a place exchange reaction can take place, through which $\mathrm{Zn}^{2+}$ is incorporated in the $\mathrm{NC}$, while $\mathrm{Cu}^{+}$or $\mathrm{In}^{3+}$ cations are extracted as $\mathrm{M}-\mathrm{R}$ species. (E) Etching: chemical species in the reaction medium can promote the partial dissolution of the CIS NC by extracting cations and/or $\mathrm{S}^{2-}$ from the lattice. (F) Homogeneous nucleation: $[\mathrm{ZnS}]$ monomers can form $\mathrm{ZnS}$ NCs through homogeneous nucleation.

semiconductor NC, the CIS shelling chemistry appears to be far more complex than that of other well-investigated systems, being seemingly intermediate to that of CdSe-based core/shell QDs, which is dominated by additive heteroepitaxial shell overgrowth with limited interfacial diffusion, ${ }^{26-31,36}$ and that of $\mathrm{PbX} / \mathrm{CdX}(\mathrm{X}=\mathrm{S}, \mathrm{Se}, \mathrm{Te})$ core/shell QDs, which is dominated by superseded shell ingrowth by topotactic $\mathrm{Pb}^{2+}$ for $\mathrm{Cd}^{2+}$ cation exchange. ${ }^{37-39}$ However, as clearly demonstrated by the variety of explanations adopted by different groups for the observed spectral blue-shifts, there is at present no consensus on the exact mechanism underlying shelling reactions on CIS NCs and on the chemical conditions required to steer the reaction toward the desired outcome.

In this work, we address the impact of precursor reactivity, reaction temperature, and surface chemistry on the delicate balance between these processes using a seeded growth approach. We demonstrate that low reaction temperatures $\left(150{ }^{\circ} \mathrm{C}\right)$ favor etching, cation exchange, and alloying, regardless of the precursors used, with the dominant process being determined by the nature of the precursors. Additive heteroepitaxial $\mathrm{ZnS}$ shell overgrowth becomes the dominant process only if reactive $S$ - and $\mathrm{Zn}$-precursors (S-ODE/OLAM and $\left.\mathrm{ZnI}_{2}\right)$ and high shelling reaction temperatures $\left(210^{\circ} \mathrm{C}\right)$ are used, although accompanied by a certain degree of heterointerfacial alloying. Remarkably, we observe that residual acetate at the surface of the CIS seed NCs favors heteroepitaxial $\mathrm{ZnS}$ shell overgrowth, while depressing alloying, etching, and cation exchange to negligible levels. This yielded, for the first time, CIS/ZnS core/shell NCs displaying red-shifted absorption spectra. Finally, we discuss the mechanisms behind the chemical processes that take place during $\mathrm{ZnS}$ shelling reactions on CIS NCs, proposing a model that explains the impact of different physical-chemical variables on the balance between these processes, ultimately dictating the outcome of the shelling reaction. These insights can be used to design synthesis strategies to CIS/ZnS core/shell and alloy NCs with tailored elemental distribution profiles, allowing precise tuning of the optoelectronic properties of the resulting materials.

\section{EXPERIMENTAL SECTION}

Materials. Copper(I) iodide (CuI, Sigma-Aldrich, 98\%), indium(III) acetate (In $(\mathrm{Ac})_{3}$, Sigma-Aldrich, 99.99\%), 1-dodecanethiol (DDT, Sigma-Aldrich $\geq 98 \%$ ), zinc stearate $\left(\mathrm{ZnSt}_{2}\right.$, Sigma-Aldrich, $10-12 \%$ Zn basis), zinc iodide (Sigma-Aldrich, 98+\%), 1-octadecene (ODE, Sigma-Aldrich, tech. 90\%), sulfur (Sigma-Aldrich 99.98\%), oleylamine (OLAM, Sigma-Aldrich, tech. 70\%), toluene (SigmaAldrich 99.8\%), methanol (Sigma Al-drich 99.8\%), 1-butanol (SigmaAldrich 99.8\%), ethanol (Sigma-Aldrich $\geq 99.8 \%$ ), acetone (VWR international, dried $\max 0.0075 \% \mathrm{H}_{2} \mathrm{O}$ ), nitric acid (Sigma-Aldrich, $65 \%)$. Prior to usage, ODE and OLAM were degassed for $2 \mathrm{~h}$ under vacuum at, respectively, 200 and $150^{\circ} \mathrm{C}$. All other chemicals were used as received.

Chalcopyrite CulnS 2 NC Synthesis. The CIS NCs were synthesized based on a protocol reported by De Trizio et al. ${ }^{14}$ Typically, CuI $(0.8 \mathrm{mmol})$ and $\mathrm{In}(\mathrm{Ac})_{3}(0.8 \mathrm{mmol})$ in $10 \mathrm{~mL}$ of DDT were mixed under inert atmosphere and degassed for $1 \mathrm{~h}$ at $80{ }^{\circ} \mathrm{C}$. Under $\mathrm{N}_{2}$ atmosphere, the temperature was raised to $210^{\circ} \mathrm{C}$, and after $40 \mathrm{~min}$ of reaction, the mixture was cooled down to room temperature. To investigate the effect of the washing protocol on the outcome of the shelling reactions, the NCs were washed by precipitation once with one of three different antisolvents: 1:1 methanol/butanol mixture, ethanol or acetone, the total volume of antisolvent being $10 \mathrm{~mL}$. The precipitate was isolated by centrifugation and redispersed in $15 \mathrm{~mL}$ of toluene.

ZnS Shelling Reactions. ZnS shelling experiments were carried out by using a seeded injection technique in which a mixture of CIS $\mathrm{NC}$ seeds and a sulfur precursor was swiftly injected under stirring into a hot solution of the $\mathrm{Zn}$-precursor in ODE at either 150 or $210{ }^{\circ} \mathrm{C}$. After $2 \mathrm{~h}$, the reaction was stopped by removing the heating source. The product NCs were washed by precipitation with isometric methanol/butanol, isolated by centrifugation, and redispersed in toluene. To study the influence of the shell precursor reactivity, a highly reactive and a less reactive precursor was used for both the cation and the anion precursors. For the $\mathrm{Zn}$-precursor, $\mathrm{Zn}(\mathrm{St})_{2}$ and $\mathrm{ZnI}_{2}\left(\Delta_{\mathrm{f}} \mathrm{H}=-208 \mathrm{~kJ} / \mathrm{mol}\right)^{40}$ were chosen as, respectively, the least reactive and the most reactive precursor. For the shelling reaction, 0.32 mmol Zn-precursor $\left(\mathrm{Zn}(\mathrm{St})_{2}\right.$ or $\left.\mathrm{ZnI}_{2}\right)$ was mixed with $4 \mathrm{~mL}$ of $\mathrm{ODE}$ and heated for $30 \mathrm{~min}$ at $100{ }^{\circ} \mathrm{C}$ before being further heated to the reaction temperature $\left(150\right.$ or $\left.210^{\circ} \mathrm{C}\right)$. As highly reactive S-precursor, elemental sulfur dissolved in ODE $\left(\Delta_{\mathrm{f}} H=0 \mathrm{~kJ} / \mathrm{mol}\right)^{40}$ was used, while DDT $\left(\Delta_{\mathrm{f}} H=-328.1 \mathrm{~kJ} / \mathrm{mol}\right)^{41}$ was chosen as the least-reactive precursor. Sulfur in ODE $(0.1 \mathrm{M})$ was prepared by dissolving $160 \mathrm{mg}$ of elemental sulfur in $50 \mathrm{~mL}$ of ODE. The mixture was heated to 200 ${ }^{\circ} \mathrm{C}$ and stirred until a clear solution was obtained. The CIS seed NCs 
( $4 \mathrm{~mL}$ of a solution of purified NCs in toluene) were sedimented by centrifugation and, depending on the sulfur precursor, redispersed in either $2 \mathrm{~mL}$ of DDT and $2 \mathrm{~mL}$ of ODE or $3 \mathrm{~mL}$ of $0.1 \mathrm{M} \mathrm{S}$-ODE and $1 \mathrm{~mL}$ of OLAM. OLAM was only added as ligand to the S-ODE solution since DDT could serve as both sulfur source and ligand. It has been shown that at high temperatures $\left(<100{ }^{\circ} \mathrm{C}\right)$ elemental sulfur reacts with OLAM (and alkylamines in general) to form $\mathrm{H}_{2} \mathrm{~S}$ and a number of reactive sulfur-containing species (e.g., alkylthioamides, dialkylamidines), ${ }^{42,43}$ which may be the actual reactive S-precursor in the reactions carried out in the present study.

Optical Characterization. Absorption spectra were recorded with a PerkinElmer lambda $950 \mathrm{UV}-$ vis/NIR spectrophotometer. PL measurements were performed with an Edinburgh Instruments FLS920 spectrofluorimeter equipped with a $450 \mathrm{~W}$ Xe lamp, a double excitation monochromator, and emission monochromator. The signal was detected with a Hamamatsu R928 PMT detector or, when the emission was at $800 \mathrm{~nm}$ or longer, with an Acton research SpectraPro 300i CCD camera with optical fiber. The magnitude of the shift of the peak positions in the optical spectra was calculated by converting the spectra to $\mathrm{eV}$ scale, according to the method reported by Ejder et al. ${ }^{44}$ and comparing the peak positions of the product NCs to the peak position of the seed CIS NCs. The peak position of the absorption spectra was determined by taking the second derivative of the spectra.

Electron Microscopy. Transmission electron microscopy (TEM) images were recorded with a Tecnai microscope (Thermo Fisher Scientific, formerly FEI company), with an acceleration voltage of 100 or $120 \mathrm{kV}$. Samples for TEM imaging were prepared by drop-casting a toluene solution of NCs onto a carbon-coated copper TEM grid. Energy dispersive X-ray spectroscopy (EDS) measurements were performed with a FEI Tecnai-20F microscope equipped with a Field Emission Gun, a Gatan 694 CCD camera and an EDAX spectrometer. The microscope was operated at $200 \mathrm{kV}$. Acquisition time for the EDS measurements was $60 \mathrm{~s}$ and for these measurements carbon-coated aluminum TEM grids were used. To ensure that the elemental concentrations were statistically valid and representative of the whole NC ensemble, EDS analyses were performed on wide areas (containing approximately $10^{4}-10^{5} \mathrm{NCs}$ ) and on triplicate (i.e., three different spots on the grid). The $\mathrm{Cu}, \mathrm{In}$, and $\mathrm{Zn}$ concentrations were determined by fitting the peaks corresponding to the K-lines. To determine the $\mathrm{Cu} / \mathrm{In}$ ratio, the average $\mathrm{Cu}$ and In concentrations $\left(C_{\mathrm{Cu}}\right.$ and $C_{\text {In }}$ ) of three measurements on the same grid were calculated (based on values $a_{\mathrm{Cu}}, b_{\mathrm{Cu}}, c_{\mathrm{Cu}}$ ). On the basis of the fit, an uncertainty percentage was given for each value, which yielded (after multiplication) a standard deviation for each concentration in each measurement (SD). The average concentrations and corresponding standard deviations were calculated as shown in the Supporting Information (page S2). High-resolution high angle annular dark field scanning TEM (HAADF-STEM) images were acquired using a cubed FEI Titan microscope operating at $300 \mathrm{kV}$. A probe semiconvergence angle of $\sim 21 \mathrm{mrad}$ was used. For electron tomography experiments, HAADF-STEM tilt series were acquired over an angular range of $\pm 75^{\circ}$ with a tilt increment of $10^{\circ}$. Because of the small size of the particles, the solution was deposited onto an ultrathin carbon support grid, which resulted in the small amount of projection images.

Inductive Coupled Plasma-Optical Emission Spectrometry (ICP-OES). Samples were prepared by drying a known volume of purified NCs in toluene. The dried NCs were dissolved in $5 \mathrm{~mL}$ of concentrated $\mathrm{HNO}_{3}$ and diluted with demineralized water. Unpurified NC solutions (i.e., crude reaction mixtures or supernatant) contain a large amount of DDT that rapidly reacts with $\mathrm{HNO}_{3}$, producing heat and hazardous gaseous products $\left(\mathrm{H}_{2} \mathrm{~S}, \mathrm{NO}_{2}\right)$, making it impossible to obtain samples suitable for ICP analysis. The samples were measured on a PerkinElmer Optima 8300 instrument and the wavelengths used for analysis were as follows: $\lambda_{\mathrm{Cu}}, 324.725 \mathrm{~nm} ; \lambda_{\mathrm{In}}, 325.609 \mathrm{~nm}$. For each concentration in each measurement, a standard deviation was given. The $\mathrm{Cu} / \mathrm{In}$ ratios and the corresponding standard deviations were calculated as explained above (see Supporting Information, page S2).

X-ray Photoelectron Spectroscopy (XPS). Samples for XPS measurements were prepared by drop casting $\mathrm{NC}$ solutions in toluene on aluminum plates, in a glovebox. Sample shipping, handling, and loading to the XPS apparatus were done under inert gas atmosphere. XP spectra were recorded on a Thermo Scientific K-Alpha spectrometer equipped with a monochromatic small-spot X-ray source and a $180^{\circ}$ double focusing hemispherical analyzer with a 128-channel delay line detector. Spectra were obtained using an aluminum anode $(\mathrm{AlK} \alpha=1486.6 \mathrm{eV})$ operated at $72 \mathrm{~W}$ and a spot size of $400 \mu \mathrm{m}$. Survey scans were measured at constant pass energy of $200 \mathrm{eV}$, and high-resolution scans of the separate regions were measured at $50 \mathrm{eV}$ pass energy. The background pressure of the ultrahigh vacuum (UHV) chamber was $<2 \times 10^{-8}$ mbar. Sample charging was compensated for by the use of an electron flood gun, and binding energy (BE) calibration was done by setting the $\mathrm{C} 1 \mathrm{~s}$ peak of $\mathrm{sp}^{3}$ carbon to $\mathrm{BE}=$ $284.8 \mathrm{eV}$. Concentrations of elements were determined by calculating the ratio of the peak areas (corrected for the relative sensitivity, electron mean free path, and transmission function). As the $\mathrm{Cu} 2 \mathrm{p}_{3 / 2}$ $(\sim 932.5 \mathrm{eV})$ and I $3 \mathrm{p}_{1 / 2}(\sim 930.8 \mathrm{eV})$ regions overlap, the fitting of the $\mathrm{Cu}$ peaks was corrected by the I concentration determined from the I $3 \mathrm{~d}_{5 / 2}$ and $\mathrm{I} 3 \mathrm{~d}_{3 / 2}$ BEs at 619.5 and $631.0 \mathrm{eV}$, respectively. We assumed a fitting uncertainty of $5 \%$ in the peak areas. This was used as standard deviation and the standard deviation in the $\mathrm{Cu} / \mathrm{In}$ ratios was calculated as explained above in the EDS paragraph (see Supporting Information, page S2).

\section{RESULTS AND DISCUSSION}

The shelling reaction procedure used in our study was the seeded injection method (see Experimental Section for details). This method was chosen to keep the CIS seed NCs as long as possible separated from heat and the precursors to minimize undesired reactions between the precursors and the CIS surface (see Figure 1). The use of trioctylphosphine (TOP) was also intentionally avoided, due to its strong binding affinity to $\mathrm{Cu}(\mathrm{I})$, which would enhance cation exchange processes by promoting the creation of $\mathrm{Cu}(\mathrm{I})$ vacancies. ${ }^{45-47}$ The chalcopyrite CIS NCs used as seeds have a trigonal pyramidal shape with an average height of $\sim 2.5 \mathrm{~nm}$ (Figure 2A), and
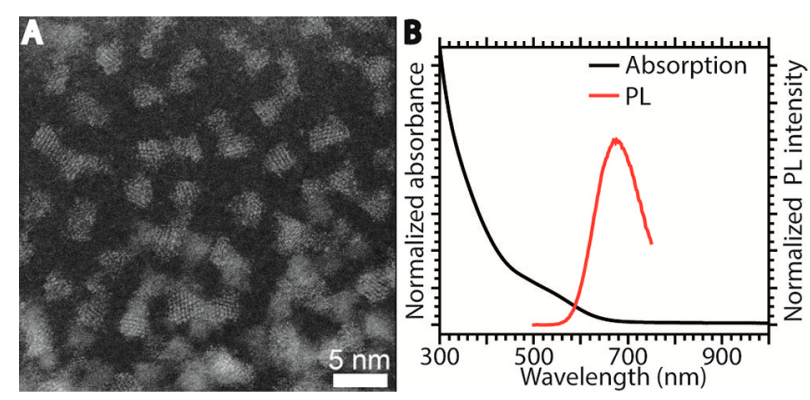

Figure 2. (A) High resolution high angle annular dark field-scanning transmission electron microscopy (HAADF-STEM) image of a representative batch of chalcopyrite CIS seed NCs. (B) Absorption and PL spectra of the CIS NCs shown in panel A.

show a featureless absorption spectrum with the lowest energy absorption centered at $530 \mathrm{~nm}$, and a broad PL peak with maximum at $676 \mathrm{~nm}$ (Figure 2B). These optical properties are in line with those typically reported for CIS NCs. ${ }^{1-6}$

Effect of Washing on Surface Chemistry. Since most processes that can take place during $\mathrm{ZnS}$ shelling reactions involve the CIS NC surface (see Figure 1 above), residual precursors and ligands that are not completely removed by the washing procedure could have a large impact on the outcome of the shelling reaction. To investigate the impact of different washing procedures on the surface chemistry of CIS NCs, a batch of freshly synthesized CIS NCs was divided into four vials 
and each portion was washed differently. Either ethanol, methanol/butanol (1:1 volume mixture), or acetone was used as antisolvent, and one portion was not washed to serve as reference. The absorption and PL spectra of the different portions after one washing cycle are shown in Figure 3. The

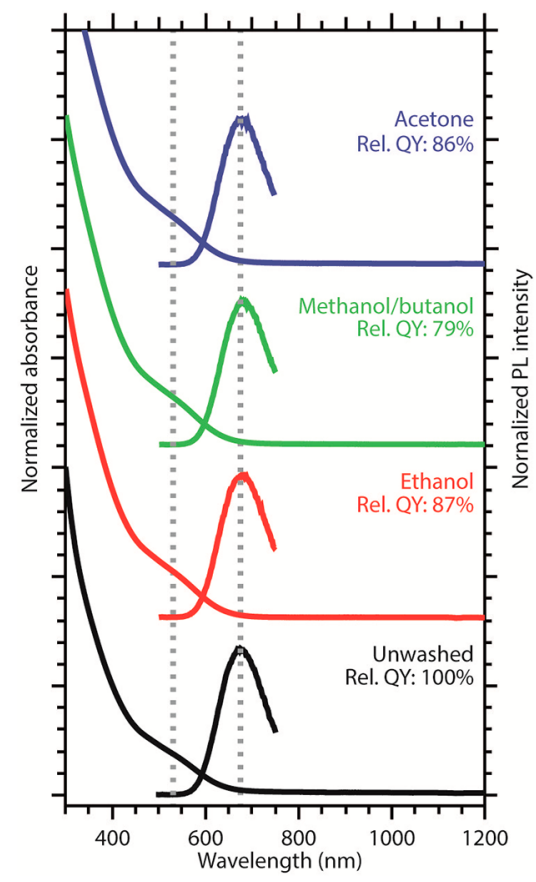

Figure 3. Absorption and PL spectra of unwashed CIS NCs (black) and CIS NCs that were washed with either ethanol (red), methanol/ butanol 1:1 mixture (green), or acetone (blue). The dotted lines mark the absorption and PL maxima. The relative PL QYs are also given. The NCs are all from the same batch and differ only with respect to the washing procedure.

dashed vertical lines indicate the peak position of the absorption and PL spectra of the unwashed CIS NCs. The key spectral features, such as the peak positions in PL and absorption spectra and PL line width, are preserved, which indicate that the electronic transitions involved are not affected by the washing procedure. If the different washing treatments had led to significant changes in elemental composition, size, or shape, this would have affected the band gap of the NCs and thus the exact peak positions. The only observed change is that the PL QY of the NCs decreased after all three washing procedures (Figure 3). This is commonly observed for colloidal NCs and is induced by partial removal of capping ligands, thereby leaving unpassivated surface sites, which act as traps for the photogenerated carriers. ${ }^{29,48}$

To verify the elemental composition of the CIS NCs, three different analysis techniques (EDS, ICP, and XPS) were used and the results are compared in Table 1 (see Experimental

Table 1. Cu/In Ratios of CIS NCs after Different Washing Procedures Determined with Three Different Techniques

$\begin{array}{lccc} & \text { EDS } & \text { ICP } & \text { XPS } \\ \text { unwashed } & 0.45 \pm 0.004 & & 0.18 \pm 0.01 \\ \text { ethanol } & 0.94 \pm 0.006 & 0.63 \pm 0.01 & 0.46 \pm 0.03 \\ \text { methanol/butanol } & 0.59 \pm 0.003 & 0.61 \pm 0.01 & 0.49 \pm 0.03 \\ \text { acetone } & 0.68 \pm 0.002 & 0.59 \pm 0.02 & 0.58 \pm 0.04\end{array}$

Section for details, representative EDS spectrum is shown in the Supporting Information Figure $\mathrm{S} 1$ ). The $\mathrm{Cu} / \mathrm{In}$ ratios of the washed samples are comparable and show only small deviations within a given technique (e.g., the ICP values vary only from 0.59 to 0.63 ). The only exception is the sample washed with ethanol, for which EDS gives a $\mathrm{Cu} / \mathrm{In}$ ratio of 0.94 , while ICP and XPS yield values that are in good agreement with those observed for the other samples using the same techniques (Table 1). The reason for this discrepancy is as yet unclear. The similarity between the $\mathrm{Cu} / \mathrm{In}$ ratios of the differently washed CIS NC samples suggests that the washing procedure does not significantly affect the elemental composition of the NCs, in agreement with the fact that the optical spectra of the CIS NCs are not observably affected by the washing procedures (Figure 3).

It is important to note that although the standard deviations for the ratios obtained for each technique can be precisely determined (see Experimental Section), the uncertainties associated with the different techniques are hard to estimate, and stem from the inherent biases associated with each technique. EDS is an electron microscopy technique in which a region on the grid is selected where mostly NCs are present. The data obtained thus reflect the elemental composition of the NCs but have an inherent uncertainty since only a small fraction of the NC ensemble can be measured at any given time. To minimize this uncertainty, we measured relatively large areas and several different spots on the grid. XPS, on the other hand, probes a larger area of a drop-casted sample, with regions of different thickness and, in case of inefficient washing, likely also different concentrations of residual ligands and unreacted precursors. If one considers the fact that the escape depth of the photogenerated electrons is limited (see below), it becomes clear that XPS may be biased toward elements in the organic matrix surrounding the NCs, if this matrix is too thick. Finally, ICP will provide the bulk composition of the sample including ligands, NCs, unreacted precursors and residual complexes formed in situ. The limitations of the different techniques are particularly critical for the unwashed sample, which cannot be analyzed by ICP (see Experimental Section), and is particularly prone to a bias toward the unreacted precursors and ligands. For this reason, one cannot meaningfully compare the $\mathrm{Cu} / \mathrm{In}$ ratios observed for the unwashed sample with those obtained for the washed samples, since they may be distorted by the presence of unreacted precursors. Nevertheless, small changes in the cation ratios as a result of the washing procedures cannot be completely excluded.

XPS is not only suitable to determine the relative concentrations of elements present in a sample, but also allows distinguishing between different chemical species of an element as the exact binding energy ( $\mathrm{BE}$ ) of electrons is determined by the oxidation state and chemical environment of the atom. For bulk materials, XPS is known as a surface sensitive technique due to the limited escape depth of the generated photoelectrons. ${ }^{49}$ However, the CIS NCs investigated here have sizes of $\sim 2.5 \mathrm{~nm}$, so the escape depth is sufficiently large to probe a substantial part of a NC, including the ligand layer. Highresolution XPS spectra of relevant elements are shown in Figure 4A-D (XPS survey spectra are shown in the Supporting Information, Figure S2, and high-resolution XPS spectra of iodide $3 \mathrm{~d}$ are shown in Figure $\mathrm{S} 3$ ). The indium $3 \mathrm{~d}_{5 / 2}$ peak is observed at a $\mathrm{BE}$ of $445.3 \mathrm{eV}$ in the unwashed sample. In the washed samples, this peak shifts to lower energies $(444.9 \mathrm{eV}$, Figure 4A), which is in good agreement with BEs earlier 

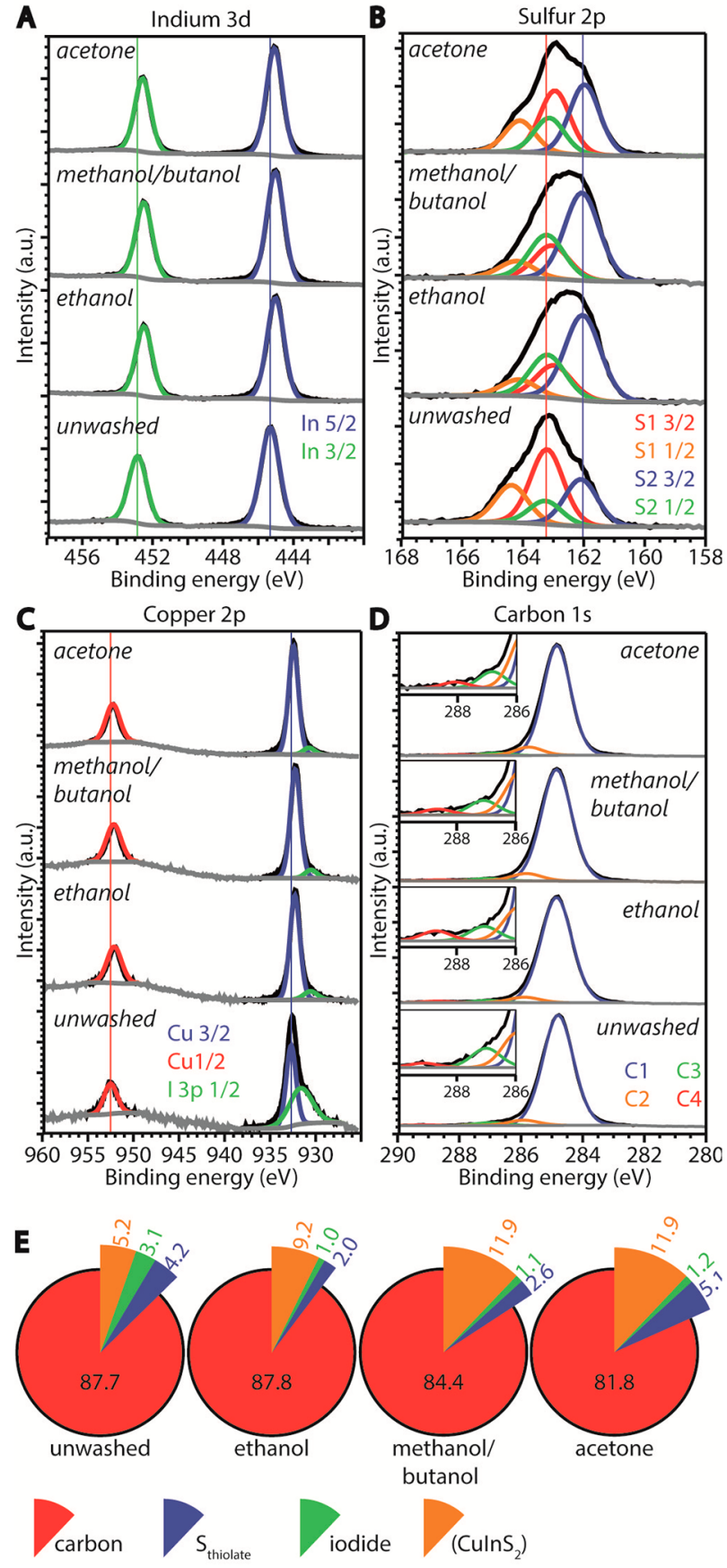

Figure 4. XPS spectra of (A) indium, (B) sulfur, (C) copper, and (D) carbon. The measured signal is shown in black, the fits to the peaks in colors, and to the background in gray. The numbers in the legends in panels $\mathrm{B}$ and $\mathrm{D}$ denote the number of different sulfur and carbon species, respectively. In the inset in panel $\mathrm{D}$, the carboxylic $\mathrm{C} 1 \mathrm{~s}$ peak at $288.6 \mathrm{eV}$ (red line) is clearly visible in the ethanol washed sample. (E) Relative concentrations of the relevant elements. The concentrations of $\mathrm{Cu}$, In, and $\mathrm{S}$ are shown together as "CuInS${ }_{2}$ ".

reported for indium in CIS NCs. ${ }^{14}$ The higher BE measured in the unwashed sample may result from a contribution of unreacted indium acetate, resulting in a convolution of peaks (the In $3 \mathrm{~d}_{5 / 2}$ peak of the similar complex indium acetylacetonate is found at a $\mathrm{BE}$ of $\left.445.6 \mathrm{eV}^{50}\right)$. However, the fwhm of the peaks (viz., 1.3 and $1.2 \mathrm{eV}$ for the unwashed and washed samples, respectively) does not justify fitting more than one peak to account for different In-containing species.
The copper $2 \mathrm{p}_{3 / 2}$ peak lies around a $\mathrm{BE}$ of $932.5 \mathrm{eV}$ and thus partially overlaps with the I $3 p_{1 / 2}$ peak around $930.7 \mathrm{eV}$. The fitting of the $\mathrm{Cu}$ peaks was therefore corrected for the I-overlap (see Experimental Section for details). In the unwashed sample, the $\mathrm{Cu} 2 \mathrm{p}_{3 / 2}$ peak has a $\mathrm{BE}$ of $932.7 \mathrm{eV}$, whereas in the washed samples this peak shifts to lower energies (932.3-932.4 eV, Figure 4C). These BEs are again in good agreement with energies reported earlier for $\mathrm{Cu}(\mathrm{I})$ in CIS NCs. ${ }^{14}$ Because the resolution in the XPS spectra is $0.2 \mathrm{eV}$ and the copper $2 \mathrm{p}_{3 / 2}$ peak in $\mathrm{CuI}$ is expected at a similar $\mathrm{BE}(932 \mathrm{eV}),{ }^{51}$ a convolution of signals cannot be excluded. The presence of $\mathrm{Cu}^{2+}$ in the samples is however excluded, as no satellite features around $942 \mathrm{eV}$ associated with divalent copper ${ }^{52,53}$ have been observed (Figure 4C). It is interesting to note that the small shifts in BEs for $\mathrm{Cu}$ and $\mathrm{In}$, between the unwashed and the washed samples, could also be ascribed to small compositional differences between the samples, as De Trizio et al. reported shifting of the $\mathrm{Cu} 2 \mathrm{p}_{3 / 2}$ peak from 932.3 to $932.6 \mathrm{eV}$ and of the In $3 \mathrm{~d}_{5 / 2}$ peak from 444.9 to $445.3 \mathrm{eV}$, upon decreasing the $\mathrm{Cu} /$ In ratio. ${ }^{14}$

The sulfur $2 p_{3 / 2}$ peaks at BEs of 163.0 (163.2 for the unwashed sample) and $162.0 \mathrm{eV}$ (Figure 4B) show that in all samples two sulfur species are present. To identify these sulfur species, we compare different literature studies on the interactions of alkanethiols with surfaces. First, thiols chemisorbed on a $\mathrm{Cu}(100)$ surface form a thiolate complex and have a sulfur $2 \mathrm{p}_{3 / 2} \mathrm{BE}$ of $162 \mathrm{eV}$, while after thermal desorption of the alkyl chain the $\mathrm{BE}$ shifts to $161.3 \mathrm{eV}$ for the remaining atomic sulfur. ${ }^{54}$ Second, on a $\operatorname{InP}(100)$ surface, the sulfur BE of alkanethiols is $162.4 \mathrm{eV}$, while for elemental sulfur at this same surface a BE of $161.6 \mathrm{eV}$ has been reported. ${ }^{55}$ Third, crystal bound DDT molecules in $\mathrm{Cu}_{2} \mathrm{~S}$ NCs give rise to $\mathrm{S} 2 \mathrm{p}_{3 / 2}$ signal at a $\mathrm{BE}$ of $161.8 \mathrm{eV}$ (sulfur as part of the crystal lattice, i.e., three-fold coordinated to $\mathrm{Cu}(\mathrm{I})$ atoms), ${ }^{56}$ while in $\mathrm{Cu}_{2} \mathrm{~S}$ NCs with surface bound DDT molecules peaks at both 161.8 and $162.2 \mathrm{eV}$ are measured, which were ascribed to sulfur as part of the crystal structure and surface-bound thiolate, respectively. ${ }^{56}$ The trend in all these studies is clear: upon adsorption, chemisorption, and subsequent desorption of the alkyl chain, leaving sulfur behind on or in the crystal, the $S 2 p_{3 / 2}$ $\mathrm{BE}$ shifts to lower values. We therefore ascribe the higher $\mathrm{BE}$ of $163.0 \mathrm{eV}$ in our study to thiolates at the surface of the NC. The slightly higher $\mathrm{BE}$ of $163.2 \mathrm{eV}$ in the unwashed sample is ascribed to the presence of DDT molecules. The peak at lower $\mathrm{BE}(162.0 \mathrm{eV})$ is attributed to sulfur ions that are part of the CIS NC, in agreement with a recent study. ${ }^{14}$ As the fwhm of the sulfur related peaks is $1.2-1.4 \mathrm{eV}$, fitting additional peaks related to other sulfur-containing species, such as free DDT or didodecylsulfide molecules, is not justified, although a recent study by Reiss and co-workers, ${ }^{57}$ reported the presence of these molecules.

Carbon species are detected in all samples and can only originate from thiolate molecules or residual acetate from the indium acetate precursor since the antisolvents used in the washing procedures have low boiling points and thus evaporate under the ultrahigh vacuum present in the XPS measurement chamber. In all samples, $\mathrm{C}$ 1s peaks are detected at BEs of $284.8,286.8-287.1$, and $285.8 \mathrm{eV}$, that we ascribe to the thiolate molecule. A fourth peak at a BE of $288.6 \mathrm{eV}$ is clearly present in the ethanol washed sample (Figure 4D). In the other samples, this peak is quite weak and is fitted in a range of 289.1 to $288.1 \mathrm{eV}$ (Figure 4D). C 1s BEs in this region are associated with esters or carboxylic groups ${ }^{58,59}$ and are in our case 
originating from the acetate group. This peak could hardly be fitted in the unwashed sample, as the relative concentration of the acetate in this sample is very low compared to that of DDT or thiolates since DDT is the reaction solvent as well. The last element detected is iodide, with a $3 \mathrm{~d}_{5 / 2} \mathrm{BE}$ of $619.8 \mathrm{eV}$ in the unwashed sample and $619.5 \mathrm{eV}$ in the washed samples and is therefore ascribed to the presence of CuI. ${ }^{51,60}$

Figure $4 \mathrm{E}$ shows the relative concentrations (see Experimental Section for details) of carbon, sulfur from thiolate, iodide, and $\mathrm{CuInS}_{2}$ (the latter obtained by adding the concentrations of $\mathrm{Cu}$, In and crystal-bound sulfur) in the four samples, as determined by XPS. In all samples, a high relative carbon concentration is measured, which is consistent with the expected carbon content (viz., 85\%), estimated based on the DDT surface coverage that has been reported by Gromova et al. (3.6 DDT molecules $\left./ \mathrm{nm}^{2}\right)^{57}$ (see Supporting Information for details). In all washed samples, the relative carbon concentration with respect to the $\mathrm{CuInS}_{2}$ concentration decreases (i.e., C/CIS $=16.9$ for the unwashed sample, 7.1 for the methanol/butanol washed sample, 6.8 for the acetone washed sample, and 9.5 for the ethanol washed sample). The relative thiolate content decreases most for the samples that are washed with ethanol or methanol/butanol (i.e., $S_{\text {thiolate }} /$ CIS is 0.8 for the unwashed sample, 0.2 for both the ethanol and methanol/butanol washed samples, and 0.4 for the acetone washed sample). Low carbon content combined with a high thiolate content, indicates efficient removal of other carbon containing species, which can only be acetate in this case. The methanol/butanol washed sample shows low carbon and low thiolate content, indicating efficient removal of both thiolates and acetate. The ethanol washed sample shows a relatively high carbon content combined with the lowest thiolate concentration, indicating residual acetate molecules. Indeed, a qualitative comparison of the acetate peaks in Figure 4D clearly shows that the acetate related $1 \mathrm{~s}$ peak is more pronounced in the ethanol washed sample compared to the other washed samples. The iodide concentration is similar in all washed samples (i.e., I/CIS is 0.1 for the washed samples and 0.6 in the unwashed sample). On the basis of these observations, we conclude that an isometric mixture of methanol and butanol is the most efficient in removing coordinating molecules (both thiolates and acetate) and that washing with ethanol leaves a significant concentration of residual acetate in the CIS NC samples.

Effect of Washing on Outcome of Shelling Reaction. The four differently washed CIS NC samples were used as seeds for shelling reactions at two different reaction temperatures (150 and $210{ }^{\circ} \mathrm{C}$ ), employing $\mathrm{ZnI}_{2}$ and S-ODE/OLAM as precursors (see Experimental Section for details). These precursors were chosen because they are both very reactive, making it less likely that the monomer formation rates dominate the reaction kinetics, thereby allowing the impact of competing reactions involving the surface (i.e., heteroepitaxial growth, alloying, etching, cation exchange, see Figure 1 above) to be more evident. Optical spectroscopy was used as main technique to investigate the outcome of the seeded shelling reactions, as shifting of the absorption peaks gives a clear indication of changes in the effective core size resulting from alloying, etching, cation exchange (blue-shift), or heteroepitaxial shell overgrowth (red-shift). We chose to use absorption rather than PL for this analysis, as there is still a lively debate on the origin of the PL in CIS NCs. ${ }^{1,2,4,12,61,62}$ It is likely that at least one localized carrier is involved in the radiative decay pathway, but the nature of this localized state is as yet unclear. ${ }^{1,4,12,61,62}$ Therefore, one can only speculate on the impact of alloying, cation exchange or shell overgrowth on this localized state and the resulting PL spectral shifts. This difficulty is further aggravated by the fact that the PL QYs of bare CIS NCs are typically very low and drastically increase after shelling reactions, therefore making the comparison between the PL spectra prior to and after shelling reactions unreliable since the PL spectrum of the CIS seed NCs may not necessarily reflect the size, shape, and composition polydispersity of the ensemble. ${ }^{2}$ In contrast, the lowest energy absorption transition is only a function of the band edge positions, which are directly related to the degree of alloying, cation exchange, or shell overgrowth.

The absorption spectra of the product NCs of all eight shelling reactions are shown in Figure 5. The spectra of the
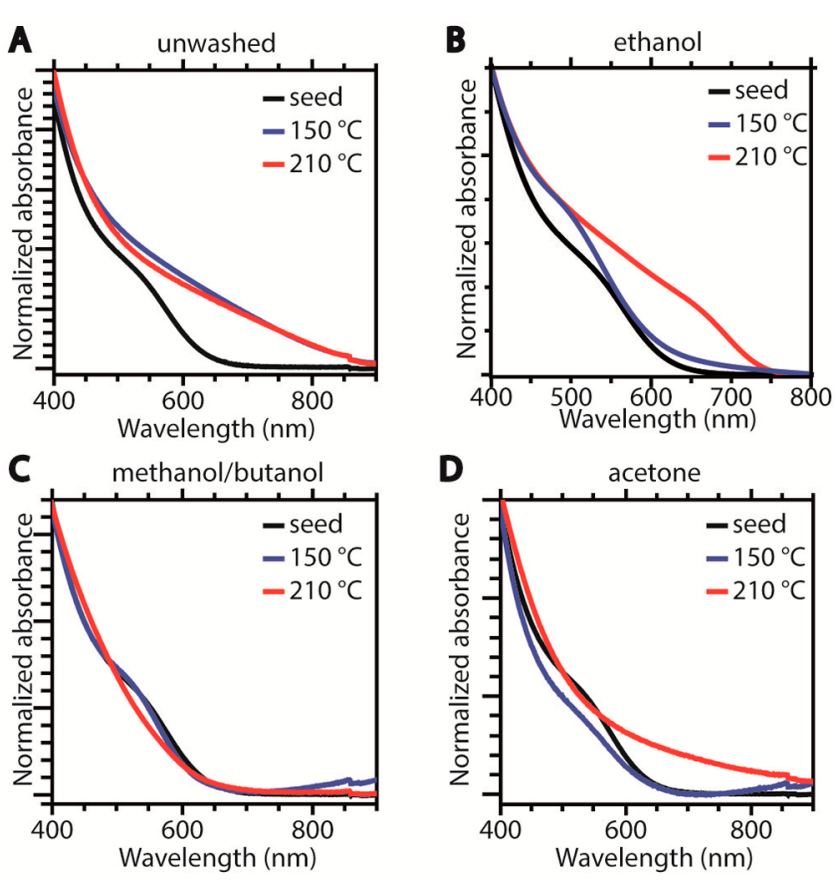

Figure 5. Absorption spectra of seed CIS NCs (black) and of product NCs after shelling reactions at $150{ }^{\circ} \mathrm{C}$ (blue) or $210{ }^{\circ} \mathrm{C}$ (red) using seeds washed in different ways: (A) unwashed, (B) ethanol washed, (C) washed with isometric methanol/butanol, (D) acetone washed.

product NCs using the unwashed core NCs are clearly redshifted with respect to the seed NCs, indicating a reduction of the quantum confinement. This observation can be explained by considering the excess of CIS precursors in the unwashed sample, which leads to further growth of the CIS NCs upon injection in the hot reaction mixture at both reaction temperatures (see Supporting Information, Figure S4). In contrast, the size of the product NCs obtained from washed CIS NCs is not significantly larger than that of the seed NCs for reactions at $150{ }^{\circ} \mathrm{C}$ but increases to $\sim 5 \mathrm{~nm}$ for reactions at $210^{\circ} \mathrm{C}$ (Figure S4), indicating the overgrowth of a $\sim 1 \mathrm{~nm}$ thick $\mathrm{ZnS}$ shell, irrespective of the washing procedure. The product NCs obtained at $210{ }^{\circ} \mathrm{C}$ using acetone washed seeds are more polydisperse and contain a larger fraction of bigger NCs $(d \geq 7$ $\mathrm{nm}$ ) than those obtained from both ethanol washed and methanol/butanol washed seeds, suggesting that ripening was more pronounced in former case, possibly due to the higher concentration of residual thiolates in the acetone washed NCs. 
These product NCs also have a lower colloidal stability than those obtained from ethanol or methanol/butanol washed seeds, which resulted in the formation of aggregates that lead to a light scattering background in the absorption spectra of these samples (see $210{ }^{\circ} \mathrm{C}$ curve in Figure 5d). Interestingly, washing with methanol/butanol or acetone resulted in spectral blueshifts at both 150 and $210^{\circ} \mathrm{C}$ (78 and $43 \mathrm{meV}$, respectively, for methanol/butanol, and 43 and $\sim 50 \mathrm{meV}$, respectively, for acetone washed samples), while the use of ethanol washed CIS seed NCs, leads to a blue-shift of $119 \mathrm{meV}$ at $150{ }^{\circ} \mathrm{C}$ and a redshift of $61 \mathrm{meV}$ after reaction at $210^{\circ} \mathrm{C}$. The latter observation is particularly relevant since in this case the observed red-shift cannot be ascribed to growth of the seed CIS NCs, as CIS coreprecursors were washed out. These results thus imply that residual acetate favors heteroepitaxial shell overgrowth at 210 ${ }^{\circ} \mathrm{C}$, thereby leading to a spectral red-shift due to exciton leakage in the shell while promoting etching and/or alloying reactions at low reaction temperatures. Moreover, these observations highlight the crucial impact of the reaction temperature on the balance between the different chemical processes taking place during the $\mathrm{ZnS}$ shelling reaction, thereby influencing not only the extent of the blue-shift induced by alloying, cation exchange, or etching reactions, but also the heteroepitaxial shell overgrowth. These inferences will be confirmed below by structural investigations. Clearly, both the reaction temperature and the surface chemistry play a crucial role in determining the balance between the different chemical processes that can take place during the shelling reaction. The interplay between these variables will be discussed in more detail in the mechanism section below after we address the impact of the precursor reactivity and investigate the atomic structure of representative $\mathrm{CIS} / \mathrm{ZnS}$ product NCs.

It should be noted that the impact of the $\mathrm{ZnS}$ shelling on the PL QYs of the CIS NCs is strongly dependent on the washing procedure, the largest enhancement being observed for unwashed seed NCs (factor 2.7 at $150{ }^{\circ} \mathrm{C}$ and 3.5 at 210 ${ }^{\circ} \mathrm{C}$ ), followed by ethanol washed and methanol/butanol washed seed NCs (factor 1 to 1.6 at $150{ }^{\circ} \mathrm{C}$ and 1.2 to 2.7 at $210{ }^{\circ} \mathrm{C}$, depending on the batch of seeds). The acetone washed seed NCs actually result in product NCs with lower PLQYs than the initial ones (reduction by a factor 3). These differences are intriguing and suggest that the state of the surface of the seed NCs is largely responsible for the quality of the CIS/ZnS heterointerface formed during the $\mathrm{ZnS}$ shelling reaction. It is also remarkable that the PL QY enhancements observed in the present work after shelling are more modest than those previously reported in the literature using similarly sized chalcopyrite CIS NCs as cores (factor 10-15). ${ }^{11,12}$ It is as yet unclear whether these differences are due to the different growth methods used (viz., seeded injection in the present work and one-pot slow addition in refs 11 and 12) or to other variables such as the nature of the shell precursors and ligands present in the reactions. Shedding light on these questions, however, is beyond the scope of this work and will be the subject of future follow-up work.

Precursor Reactivity. The results discussed above show that both the reaction temperature and the presence of residual acetate and thiolate in the CIS seed NCs have a dramatic impact on the outcome of the shelling reaction. Another crucial variable is the precursor reactivity, which determines the monomer formation rate and therefore directly impacts on the kinetics of both the heteroepitaxial growth and the homogeneous secondary nucleation (Figure 1). ${ }^{29,36,63-67}$ Further, the precursor reactivity also affects the rates of undesired parallel processes such as cation exchange, etching, and alloying (Figure 1). Methanol/butanol washed CIS NCs were used as seeds to investigate the influence of the precursor reactivity since this washing was the most effective in removing coordinating molecules (both acetate and thiolate, see above), therefore minimizing possible synergistic or antagonist interactions with the precursors, allowing the isolated effect of the precursor reactivity to be more clearly observed. It should be noted that the precursor reactivity is determined by both its intrinsic stability and the reaction temperature. Therefore, also in this case two different shelling reaction temperatures were used. The outcome of the shelling reactions was followed not only by the extent of the spectral shifts observed for the product NCs, but also by the change in their elemental composition with respect to the CIS seed NCs (Figure 6 and Supporting

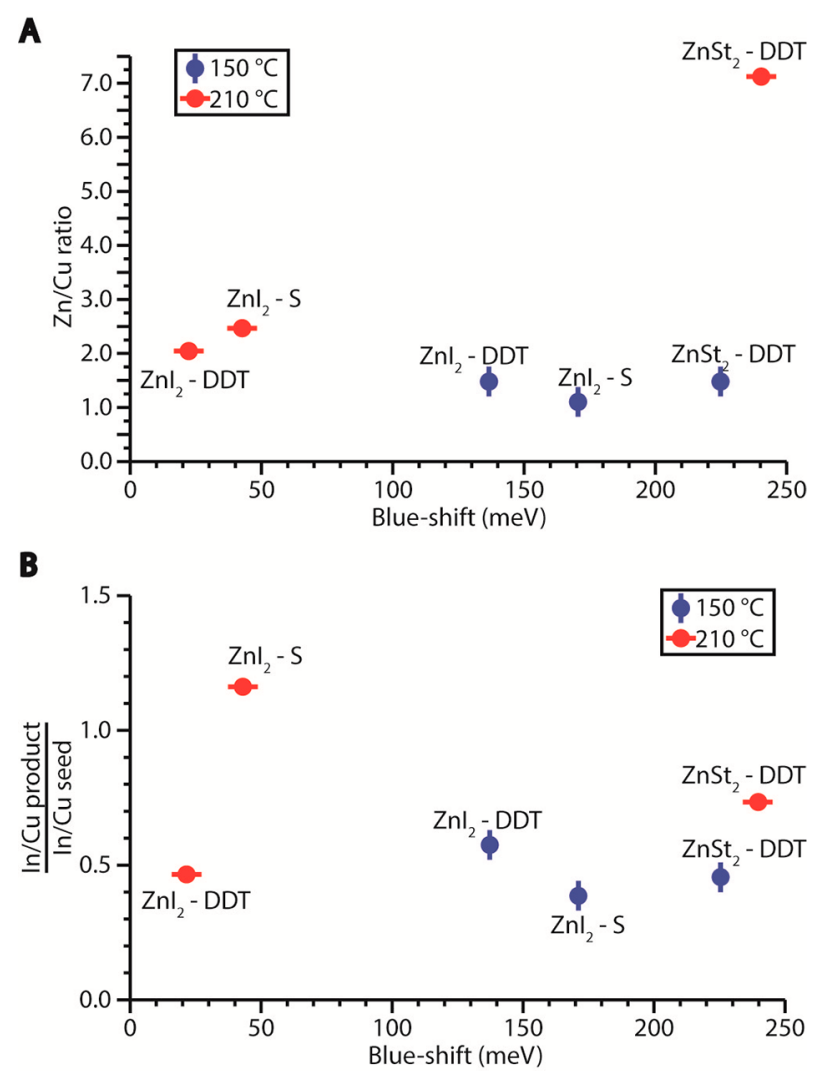

Figure 6. (A) Blue-shift in absorption spectra and $\mathrm{Zn} / \mathrm{Cu}$ ratio of product NCs after shelling reactions using methanol/butanol washed CIS NCs as seeds and different precursor combinations and reaction temperatures. (B) Blue-shift in absorption spectra with respect to the change in the $\mathrm{In} / \mathrm{Cu}$ ratio after the shelling reaction for the same samples shown in panel A. The change in the $\mathrm{In} / \mathrm{Cu}$ ratio is obtained by dividing the ratios observed for the product NCs by those observed for the seed NCs.

Information Figure S5). Taken together, these variables allow the dominant chemical processes acting on the CIS seed NCs during the $\mathrm{ZnS}$ shelling reactions to be identified.

The data corresponding to the product NCs obtained from the reaction using $\mathrm{ZnSt}_{2}$ and $\mathrm{S}-\mathrm{ODE}$ as precursors is not included in Figure 6, since in this case large 2D nanoplatelets and nanosheets were obtained at both reaction temperatures (Figure S6). This intriguing outcome has been studied in detail in previous work by our group ${ }^{43}$ and shown to be induced by 
$\mathrm{Znl}+2+\mathrm{S}-\mathrm{ODE}, 150^{\circ} \mathrm{C}$

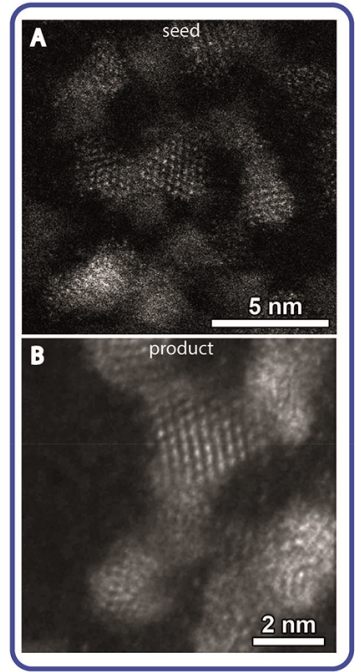

$\mathrm{Znl} \mathrm{S}_{2}+\mathrm{S}-\mathrm{ODE}, 210^{\circ} \mathrm{C}$

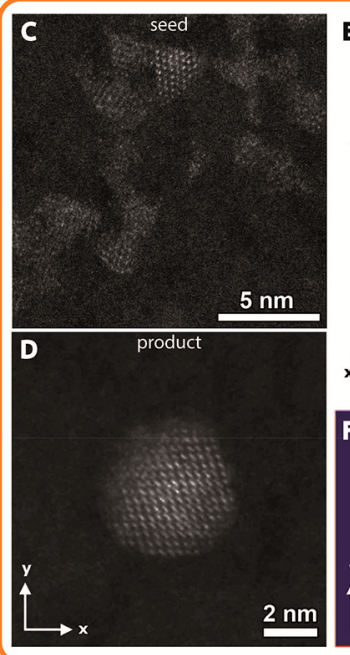

Figure 7. (A) High resolution HAADF-STEM image of (ethanol washed) CIS seed NCs. The NCs have a trigonal pyramidal shape with an average base of $2.7 \pm 0.3 \mathrm{~nm}$ and an average height of $2.5 \pm 0.3 \mathrm{~nm}$. (B) High resolution HAADF-STEM image of product NCs obtained after shelling reaction using the CIS NCs shown in panel A as seeds at $150^{\circ} \mathrm{C}$. The average size of the product NCs is $3.2 \pm 0.7 \mathrm{~nm}$ for the base and $3.0 \pm 0.4 \mathrm{~nm}$ for the height. (C) High resolution HAADF-STEM image of (ethanol washed) CIS seed NCs from a different batch with respect to those shown in panel A. The NCs have a trigonal pyramidal shape with an average base of $2.5 \pm 0.3 \mathrm{~nm}$ and an average height of $2.3 \pm 0.3 \mathrm{~nm}$. (D) High resolution HAADF-STEM image of product NC obtained after shelling reaction using the CIS NCs shown in panel C as seeds at $210^{\circ} \mathrm{C}$. The average diameter of the product NCs in this sample is $4.7 \pm 0.8 \mathrm{~nm}$. (E) 3D visualization of a product CIS/ZnS NC from the same sample shown in panel D with indication of the position of the orthoslices shown in panels $\mathrm{F}$ and $\mathrm{G}$. The inset shows the 3D visualization with the location of the core indicated in magenta. (F, G) Orthoslices through the 3D reconstruction shown in panel E (intensity is color-coded, increasing from blue to red in the same sequence as in the visible spectrum). Additional HR HAADF-STEM images are shown in the Supporting Information (Figure S7).

fast cation-extraction by in situ generated reactive sulfurcontaining species (e.g., $\mathrm{H}_{2} \mathrm{~S}$ ). This process converts the CIS seed NCs into In-poor CIS NCs, which subsequently undergo $2 \mathrm{D}$ self-organization and oriented attachment, yielding In-poor CIS nanosheets. ${ }^{43}$ This demonstrates that S-ODE/OLAM is capable of quickly extracting $\mathrm{In}^{3+}$ (and to a lesser extent $\mathrm{Cu}^{+}$) from CIS NCs, thereby leading to pronounced etching, which is characterized by large blue-shifts accompanied by large reductions in the $\mathrm{In} / \mathrm{Cu}$ ratio and low $\mathrm{Zn}$-content. The extent of the etching is directly related to the reactivity of the $\mathrm{Zn}$ precursor: an unreactive $\mathrm{Zn}$-precursor, such as $\mathrm{ZnSt}_{2}$, is unable to react with the $S$-precursors fast enough to outcompete the In-extraction reaction, thereby resulting in pronounced etching, followed by $2 \mathrm{D}$ self-organization. In contrast, a reactive precursor, such as $\mathrm{ZnI}_{2}$ at $210{ }^{\circ} \mathrm{C}$, can effectively prevent $\mathrm{NC}$ etching by quickly reacting with the $\mathrm{S}$-precursors to form $[\mathrm{ZnS}]$ monomers, which in turn favors heteroepitaxial shell overgrowth, leading to relatively small blue-shifts and high $\mathrm{Zn}$ content, accompanied by unchanged (or slightly increased) In/ $\mathrm{Cu}$ ratios (Figure 6). Interestingly, decreasing the reaction temperature to $150{ }^{\circ} \mathrm{C}$ has a higher impact on the reactivity of $\mathrm{ZnI}_{2}$ (and thereby on the $[\mathrm{ZnS}]$ formation rate) than on the reactivity of S-ODE/OLAM, thereby shifting the balance toward the NC etching reaction, resulting in larger blue-shifs, lower $\mathrm{Zn}$-content, and larger decrease in the $\mathrm{In} / \mathrm{Cu}$ ratio, with respect to the reaction carried out at $210{ }^{\circ} \mathrm{C}$.

The data in Figure 6 show that shelling reactions at $150{ }^{\circ} \mathrm{C}$ (blue data points) invariably lead to larger blue-shifts, lower $\mathrm{Zn} / \mathrm{Cu}$ ratios, and larger reductions in $\mathrm{In} / \mathrm{Cu}$ with respect to the same reaction carried out at $210^{\circ} \mathrm{C}$. This shows that lower reaction temperatures favor etching, alloying, and cation exchange. $\mathrm{Zn}^{2+}$ for $\mathrm{Cu}^{+}$or $\mathrm{In}^{3+} \mathrm{CE}$ is favored by using more stable precursors, such as $\mathrm{Zn}(\mathrm{St})_{2}$ and DDT, since in this case the precursor to monomer conversion rate will be slow, leaving the $\mathrm{Zn}$-precursor available to bind to the NC surface and undergo cation exchange. This process is further favored in the presence of DDT because this molecule is unable to induce extensive etching, being already the capping ligand shell on CIS NCs synthesized with DDT as S-source, ${ }^{57}$ thereby leaving a relatively stable surface for adsorption of the $\mathrm{Zn}$-precursor. The prevalence of CE under these conditions is clearly evidenced by the observation that the shelling reaction using $\mathrm{Zn}(\mathrm{St})_{2}$ and DDT at $210{ }^{\circ} \mathrm{C}$ leads to the largest blue-shift and the highest $\mathrm{Zn}$-content. Moreover, replacing $\mathrm{Zn}(\mathrm{St})_{2}$ by the more reactive $\mathrm{ZnI}_{2}$ leads to smaller blue-shifts and lower $\mathrm{Zn}$-contents. This is in line with the expected trend in the CE efficiencies since the driving force for the $\mathrm{CE}$ reaction is the energy gain upon replacing the native cation by the guest cation. ${ }^{47,68}$ Considering that $\mathrm{In}^{3+}$ is a hard Lewis acid, ${ }^{69}$ the formation of In(III) stearate is more favorable than that of $\operatorname{In}(\mathrm{III})$ iodide, as stearate is a harder Lewis base and stronger ligand than iodide. This also explains why $\mathrm{Zn}^{2+}$ for $\mathrm{In}^{3+} \mathrm{CE}$ is more efficient than that of $\mathrm{Zn}^{2+}$ for $\mathrm{Cu}^{+}{ }^{14}$ It is thus clear that the outcome of a $\mathrm{ZnS}$ shelling reaction on CIS NCs is determined by a delicate balance between the rates of all the chemical processes depicted in Figure 1 and that this balance is highly sensitive not only to the reaction temperature and the nature of the $\mathrm{Zn}$ - and $\mathrm{S}$ precursors, but also to the presence of residual coordinating molecules at the surface of the NCs. The mechanism behind this complex interplay of physical-chemical processes will be discussed in more detail below after the atomistic structure characterization of two representative examples of product NCs.

Structural Characterization. The observations described above suggest that the use of reactive $\mathrm{S}$ - and $\mathrm{Zn}$-precursors $\left(\mathrm{ZnI}_{2}\right.$ and $\left.\mathrm{S}-\mathrm{ODE} / \mathrm{OLAM}\right)$ at high temperatures $\left(210^{\circ} \mathrm{C}\right)$ in 
the presence of acetate leads to heteroepitaxial $\mathrm{ZnS}$ shell overgrowth on CIS NCs, with negligible contribution of undesired competing processes such as $\mathrm{CE}$, alloying, and etching, while low reaction temperature $\left(150^{\circ} \mathrm{C}\right)$ or unreactive precursors shift the balance toward the competing processes. To verify whether this is indeed the case, the structure and composition of the seed and product NCs were investigated with transmission electron microscopy. Figure 7A and $\mathrm{C}$ show high-resolution (HR) HAADF-STEM images of CIS NCs, washed with ethanol and used as seeds for a shelling reaction with S-ODE/OLAM and $\mathrm{ZnI}_{2}$ at 150 or $210^{\circ} \mathrm{C}$, respectively. In Figure $7 \mathrm{~B}$ and D, HR-HAADF-STEM images of product NCs obtained from these reactions are shown. The absorption spectrum of the product NCs obtained at $150{ }^{\circ} \mathrm{C}$ is blue-shifted by $40 \mathrm{meV}\left([\mathrm{In} / \mathrm{Cu}]_{\text {product } / \text { seed }}=0.5\right)$, while that of the product NCs obtained at $210{ }^{\circ} \mathrm{C}$ is red-shifted by $200 \mathrm{meV}$ ([ $\mathrm{In} /$ $\left.\mathrm{Cu}]_{\text {product/seed }}=1.0\right)$. It is immediately clear that the shape of the seed NCs (trigonal pyramid) is essentially preserved in the product NCs obtained at $150{ }^{\circ} \mathrm{C}$, despite a small growth (base increases from $2.7 \pm 0.3 \mathrm{~nm}$ to $3.2 \pm 0.7 \mathrm{~nm}$ and height increases from $2.5 \pm 0.3 \mathrm{~nm}$ to $3.0 \pm 0.4 \mathrm{~nm}$ ). In contrast, the product NCs obtained at $210{ }^{\circ} \mathrm{C}$ have a different shape and a significantly larger size (average diameter of $4.7 \pm 0.8 \mathrm{~nm}$ ) with respect to the seed NCs (trigonal pyramids with an average base of $2.5 \pm 0.3 \mathrm{~nm}$ and an average height of $2.3 \pm 0.3 \mathrm{~nm}$ ). Moreover, the NCs obtained at $150{ }^{\circ} \mathrm{C}$ (Figure $7 \mathrm{~B}$ ) show a uniform intensity over the whole $\mathrm{NC}$, indicating that they consist of $(\mathrm{Cu}, \mathrm{In}, \mathrm{Zn}) \mathrm{S}_{2}$ alloys. This is consistent with the observation of blue-shifted spectra after the reaction, as discussed above. In contrast, the NCs obtained at $210{ }^{\circ} \mathrm{C}$ (Figure 7D) show increased intensity in the center of the particle, suggesting that either the heavier elements are mostly located in the center or an increased thickness is present at the center of the NCs. Since the intensity in HAADF-STEM images scales with both the projected thickness and the atomic number of the present elements, such $2 \mathrm{D}$ images cannot unambiguously confirm core-shell architectures. Therefore, electron tomography was applied to study the internal structure of the CIS/ZnS product NCs. Figure $7 \mathrm{E}$ shows the 3D visualization of a CIS/ZnS core/shell $\mathrm{NC}$ investigated during the electron tomography experiment. In Figure $7 \mathrm{~F}$ and G, 2D slices through the $3 \mathrm{D}$ reconstruction are shown, with indication of the position of the slices in Figure 7E. In Figure 7G, a more intense region (red color) is detected, compared to the orthoslice in Figure 7F. Since indium has a much larger atomic mass $\left(Z_{\mathrm{In}}=49\right)$ than the other elements present $\left(Z_{\mathrm{Cu}}=29\right.$ and $Z_{\mathrm{Zn}}=30$ ), its presence in the core of the NC is confirmed, while the surface is richer in the lighter elements copper and/or zinc. From these tomography results, we conclude that the $\mathrm{ZnS}$ shell grows anisotropically. This is similar to previous observations on wurtzite $\mathrm{CdSe} / \mathrm{CdS}$ dot core/rod shell nanorods ${ }^{46,70-72}$ and nanobullets $^{36}$ and wurtzite graded $\mathrm{AgInSe}_{2} / \mathrm{ZnSe}$ core/shell $\mathrm{NCs},{ }^{73}$ suggesting that the facets of the trigonal pyramidal shaped CIS seed NCs are not equivalent. However, the CIS seed NCs and the product NCs (both the alloy NCs obtained at $150{ }^{\circ} \mathrm{C}$ and the $\mathrm{CIS} / \mathrm{ZnS}$ core/shell NCs obtained at 210 ${ }^{\circ} \mathrm{C}$ ) have the chalcopyrite structure (Supporting Information, Figure S7), which, in contrast with the hexagonal wurtzite structure, does not possess polar facets. Therefore, the anisotropy observed in the heteroepitaxial shell overgrowth at $210{ }^{\circ} \mathrm{C}$ may be attributed to a different termination of the pyramid base with respect to the sides, either due to a different facet composition or due to selective adhesion of acetate, or a combination of both.

Mechanism. The results discussed above provide valuable insights on the chemical processes that take place during $\mathrm{ZnS}$ shelling reactions on CIS NCs (Figure 1), allowing us to understand the mechanisms behind them and the factors that determine the balance between these processes, ultimately dictating the outcome of the shelling reaction and the optoelectronic properties of the product NCs. These processes can be roughly divided into three categories: (i) reactions taking place in solution, such as the precursor to $[\mathrm{ZnS}]$ monomer conversion, homogeneous nucleation of $\mathrm{ZnS}$ NCs, and reactions involving the added precursors or ligands forming new species in situ (e.g., reaction between elemental Sulfur and alkylamines forming $\mathrm{H}_{2} \mathrm{~S}$, alkylthioamides, dialkylamidines ${ }^{42}$ ); (ii) reactions with the surface (i.e., reactions that alter the chemical composition of the NC facets), such as etching, cation exchange, and alloying; and (iii) reactions on the surface, such as additive heteroepitaxial shell overgrowth and ligand adsorption or desorption. These reactions may occur in parallel, competing with each other for the limited supply of precursors, monomers, or available surface sites (e.g., etching, cation exchange, and alloying compete with heteropitaxial shell growth), or sequentially, in a concerted manner (e.g., monomer formation precedes both heteroepitaxial shell growth and homogeneous nucleation). Therefore, the outcome of the reaction depends on a complex interplay between a number of inherently linked elementary kinetic steps.

The precursor to monomer conversion has been shown to be the rate-limiting step in the formation of a variety of binary metal chalcogenides (viz., $\mathrm{CdX}, \mathrm{PbX}, \mathrm{Cu}_{2} \mathrm{~S}, \mathrm{X}=\mathrm{S}, \mathrm{Se}$ ), ${ }^{29,63-66}$ and is likely also rate-limiting in heteroepitaxial shell overgrowth reactions, ${ }^{29,36,67}$ since recent work has suggested that heteroepitaxial shells grow by incorporation of monomer units, rather than by layer-by-layer adsorption of atomic species, ${ }^{29,67}$ even when the SILAR strategy is employed. ${ }^{36}$ The adsorption of atomic species or precursors is in fact more likely to lead to etching, since the adsorbed species may leave again after reacting with the surface site, thereby carrying a metal or chalcogen atom with itself, before more precursors can join in to form a sufficiently stable adlayer. Etching can also be promoted by ligands or reactive species formed in situ. Alternatively, the adsorbed metal precursor may lead to cation exchange (CE). Nanoscale CE has been extensively investigated in recent years as a postsynthetic strategy to control the composition of NCs, and is thus well-understood. ${ }^{47}$ The CE process itself is essentially a surface reaction, which would be self-limited in the absence of cation diffusion in the NC, stopping as soon as all surface native cations had been exchanged by guest cations. ${ }^{68}$ Therefore, solid state diffusion fluxes must be set in motion to allow the reaction to proceed. Depending on the solid state diffusion rates of the guest and host cations, the $\mathrm{CE}$ process can lead to either superseded shell ingrowth (e.g., $\mathrm{PbSe} / \mathrm{CdSe}, \mathrm{ZnSe} / \mathrm{CdSe})^{37-39,68}$ or alloy NCs (graded or homogeneous, e.g., $(\mathrm{Zn}, \mathrm{Cd}) \mathrm{Se}^{68} \mathrm{CuInS}_{2}$ from $\mathrm{Cu}_{2-x} \mathrm{~S},{ }^{45}$ (Cu,In,Zn)S ${ }_{2}{ }^{14}{ }^{C} \mathrm{CsPBr}_{3}: \mathrm{M}$ with $\mathrm{M}=\mathrm{Sn}^{2+}, \mathrm{Cd}^{2+}$, $\left.\mathrm{Zn}^{2+74}\right)$. Diffusion may lead to alloying, even in the absence of cation exchange since $\mathrm{Zn}^{2+}$ cations from $[\mathrm{ZnS}]$ monomers deposited at the surface may diffuse in the CIS NCs if a sufficiently stable $\mathrm{ZnS}$ heteroepitaxial monolayer is not formed fast enough. This is expected because the binding strength of a $\mathrm{Zn}^{2+}$ in an isolated $[\mathrm{ZnS}]$ adsorbate is much weaker than that in 
a fully grown $\mathrm{ZnS}$ shell due to its lower coordination number, resulting in much lower activation energies for diffusion.

Each of the processes described above has its own activation energy, and therefore depends differently on temperature. Consequently, the reaction temperature has a dramatic impact on the balance between the rates of the different processes, and can thus be used as a sensitive parameter to tailor the product of shelling reactions. The observations discussed above show that low temperatures $\left(150{ }^{\circ} \mathrm{C}\right)$ favor etching, alloying, and cation exchange, leading to pronounced spectral blue-shifts. This can be rationalized by considering that at this temperature the $[\mathrm{ZnS}]$ monomer formation rates are too low, and therefore, heteroepitaxial overgrowth by monomer addition is unable to outcompete the other processes. The unreacted shell precursors are then available to undergo direct reactions with accessible sites at the surface of the CIS NCs, reducing the monomer formation rates even further. The dominant process under these conditions will then depend on the nature of the precursors. Reactive S-precursors (S-ODE/OLAM) combined with unreactive $\mathrm{Zn}$-precursors $\left(\mathrm{ZnSt}_{2}\right)$ lead primarily to etching, resulting in product NCs with large spectral blueshifts $(>150 \mathrm{meV})$ and low Zn-content, while the use of reactive $\mathrm{S}$ - and $\mathrm{Zn}$-precursors (S-ODE/OLAM and $\mathrm{ZnI}_{2}$ ) results in a combination of etching and $[\mathrm{ZnS}]$ deposition followed by interdiffusion, yielding product (CIS, $\mathrm{ZnS}$ ) alloy NCs with intermediate blue-shifts (50-150 meV), high $\mathrm{Zn}$ content, and decreased $\mathrm{In} / \mathrm{Cu}$ ratio with respect to the seed NCs.

Conversely, unreactive S-precursors (DDT) favor cation exchange involving the Zn-precursors, which is most efficient when the $\mathrm{Zn}$-precursor is also less reactive $\left(\mathrm{ZnSt}_{2}\right)$. As discussed above, this is due to the fact that the driving force for $\mathrm{CE}$ reactions is the energy gain upon replacement of the native cation by the guest cation, ${ }^{47,68}$ which is larger if the $\mathrm{Zn}^{2+}$ precursor is more stable since in this case the leaving $\mathrm{In}^{3+}$ complex will be more stable due to the hard Lewis acid nature of $\mathrm{In}^{3+}{ }^{69}$ Therefore, increasing the reaction temperature (210 $\left.{ }^{\circ} \mathrm{C}\right)$, while using a combination of unreactive precursors $\left(\mathrm{ZnSt}_{2}\right.$ and DDT), increases the CE rates even further, leading to large spectral blue-shifts $(>150 \mathrm{meV})$ and high Zn-contents. In contrast, high reaction temperatures and reactive precursors shift the balance toward the $[\mathrm{ZnS}]$ monomer formation, thereby boosting the heteroepitaxial growth rates, while depressing the rates of the competing processes. As a result, heteroepitaxial shell overgrowth dominates, leading to product NCs with high Zn-content, small spectral blue-shift $(<100$ $\mathrm{meV}$ ), and minimally changed $\mathrm{In} / \mathrm{Cu}$ ratios. The relatively small blue-shifts indicate that a certain degree of $\mathrm{ZnS}$ interdiffusion and alloying still occurs, presumably at the early stages of the shell overgrowth.

From this perspective, it is remarkable that the presence of acetate in the ethanol washed CIS seed NCs is capable of completely suppressing the spectral blue-shifts, leading instead to CIS/ZnS core/shell NCs displaying spectral red-shifts (60$200 \mathrm{meV}$ ). It is interesting to note that addition of zinc acetate to the shelling reaction mixture results in a pronounced spectral blue-shift (Supporting Information, Figure S8), suggesting that its lower reactivity with respect to $\mathrm{ZnI}_{2}\left(\Delta_{\mathrm{f}} \mathrm{H}=-1669 \mathrm{~kJ} / \mathrm{mol}\right.$ and $-208 \mathrm{~kJ} / \mathrm{mol}$, for $\mathrm{Zn}(\mathrm{Ac})_{2} \cdot \mathrm{H}_{2} \mathrm{O}^{75}$ and $\mathrm{ZnI}_{2},{ }^{40}$ respectively) leads again to a dominance of etching by reactive $S$-species and $[\mathrm{ZnS}]$ interdiffusion. This demonstrates that acetate should already be present prior to the onset of the shelling reaction to facilitate the heteroepitaxial $\mathrm{ZnS}$ growth, suggesting that it exerts its influence by modulating the surface availability and stability. Reactions with the surface (etching, CE, alloying) result from an accessible and dynamic surface, while a reaction on the surface, such as heteroepitaxial overgrowth, requires an accessible but steady surface. We thus propose that acetate at the surface of the CIS seed NCs will stabilize its surface and hinder access to active sites until the concentration of $[\mathrm{ZnS}]$ monomers in solution is sufficiently high to lead to heteroepitaxial growth rates that are fast enough to outcompete the $\mathrm{Zn}^{2+}$ interdiffusion and other competing processes. In other words, the release rate of acetate from the surface of the CIS seed NCs is sufficiently slow to allow the concentration of [ZnS] monomers to build-up, while preventing unreacted precursors and other active species from binding at active surface sites, thereby inhibiting etching and cation exchange, since binding of the $\mathrm{Zn}$-precursor or etchant species to the surface is the first step in these processes. ${ }^{68,76}$ On the basis of the observation of anisotropic shell growth (see above), it is likely that acetate is more strongly bound to one facet in particular.

It should be noted that homogeneous nucleation of $\mathrm{ZnS} \mathrm{NCs}$ was not observed in any of the experiments discussed above. This can be attributed to the fact that the activation energies for homogeneous nucleation are even higher than those for heterogeneous nucleation and heteroepitaxial overgrowth. ${ }^{29}$ Therefore, homogeneous nucleation would only be significant if the monomer formation rates would exceed the heteroepitaxial growth rates or if the NC surface would not be accessible to the $[\mathrm{ZnS}]$ monomers due to a too dense and strongly bound ligand layer, which was clearly not the case under the conditions prevalent in our study.

The insights obtained in our work can be used to design synthesis strategies to CIS/ZnS core/shell and alloy NCs and are also useful to rationalize the seemingly disparate results presented in the literature, with reported spectral blue-shifts ranging from 60 to $340 \mathrm{meV}$. $2,11-14,18,23,24,32-35$ The diversity of the trends reported in literature for $\mathrm{ZnS}$ shelling reactions on CIS NCs can be understood in light of the mechanisms proposed in our study and can be ascribed to the wide variety of reaction conditions used. Most studies used the dropwise addition of relatively unreactive shell precursors (typically $\mathrm{ZnSt}_{2}$ and TOP-S $)$ to a hot $\left(T \geq 200{ }^{\circ} \mathrm{C}\right)$ solution containing CIS NCs. ${ }^{2,11-14,18,23,24,32-35}$ Such conditions will indisputably favor alloying since the $[\mathrm{ZnS}]$ monomer formation rates will be slow and the surface mobility will be high. The presence of TOP in many studies ${ }^{2,11,12,14,76}$ will also favor cation exchange and etching because TOP (a soft Lewis base with absolute hardness $\eta \approx 6 \mathrm{eV}),{ }^{77}$ has a strong affinity for $\mathrm{Cu}^{+}$(a soft Lewis acid with $\eta=6.28 \mathrm{eV}),{ }^{69}$ and also for sulfur, thereby being able to extract both from the CIS NCs. Several studies have also used the crude reaction mixture (so unwashed CIS seed NCs), ${ }^{12,14,15,24,25,33-35}$ thereby adding residual $\mathrm{Cu}$ - and Inprecursors to the shelling reaction mixture. This should facilitate the formation of (gradient) alloy NCs even further, since the deposition and interdiffusion of $[\mathrm{ZnS}]$ monomers would be accompanied by incorporation of both $\mathrm{Cu}$ and $\mathrm{In}$. Considering that the magnitude of the spectral blue-shift depends on the extent of the $\mathrm{Zn}$ interdiffusion and alloying (and on the extent of prior etching of the NC seeds), one can easily see that differences in the reaction temperatures and reaction times used in different studies will also add to the spread in the observed blue-shifts. 
Interestingly, the crystal structure of the CIS seed NCs is not a relevant parameter from this viewpoint since a recent study by our group has shown that the spectral blue-shifts observed after $\mathrm{ZnS}$ shelling of wurtzite CIS NCs by dropwise addition of $\mathrm{Zn}$ and S-precursors (viz., 50-150 meV, with unchanged $\mathrm{In} / \mathrm{Cu}$ ratio) are analogous to those observed for chalcopyrite CIS NCs under similar conditions. ${ }^{2}$ However, under seeded growth conditions, the crystal structure of the CIS seed NCs has a dramatic impact on the morphology of the product CIS/ZnS hetero-NCs, leading to CIS/ZnS dot core/rod shell heteronanorods, if wurtzite CIS NCs are used as seeds and the [ $\mathrm{ZnS}$ ] monomer concentration is allowed to build-up by delaying the injection of the seeds with respect to the injection of the $\mathrm{Zn}$ and S-precursors. ${ }^{78}$ This recent study clearly demonstrates the crucial role of the monomer formation rates in determining the outcome of shelling reactions, in agreement with the mechanism proposed above. There are also studies that used conditions that favor cation exchange (e.g., addition of only $\mathrm{Zn}$ precursors), resulting in (CIS, ZnS) alloy NCs with high $\mathrm{Zn}$ content and consequently very large blue-shifts $(340 \mathrm{meV}) .{ }^{14}$ In this context, the recent study by Woods and co-workers on the preparation of CISe/ $\mathrm{ZnSe}_{1-x} \mathrm{~S}_{x}$ alloyed core/shell NCs by $\mathrm{Zn}^{2+}$ for $\mathrm{In}^{3+}$ and $\mathrm{Cu}^{+}$cation exchange is particularly interesting. ${ }^{76}$ The authors demonstrated that the thickness of the ingrown superseded shell was determined by the reaction temperature (lower temperatures yielding thinner shells), concluding that the diffusion of $\mathrm{Zn}^{2+}$ cations into successive atomic monolayers of the seed NCs is a thermally activated process in which the activation energy increases in a depthdependent fashion. ${ }^{76}$ Similar observations have been reported before for $\mathrm{ZnSe} / \mathrm{CdSe}$ core/shell and graded alloy $\mathrm{NCs}{ }^{68}$ demonstrating that the reaction temperature is a sensitive parameter to tailor the composition and the elemental distribution profile of semiconductor hetero-NCs from core/ shell to homogeneous alloy NCs through graded alloys, thereby tuning the carrier localization regime and the optoelectronic properties of the resulting materials.

\section{CONCLUSIONS}

The outcome of $\mathrm{ZnS}$ shelling reactions on CIS NCs is determined by a delicate balance between several chemical processes that take place both sequentially and in parallel, competing with each other for the limited supply of shell precursors and available surface sites. In this study, we investigated the impact of the precursor reactivity, reaction temperature, and surface chemistry on the outcome of the shelling reaction using a seeded growth strategy.

We demonstrate that low reaction temperatures $\left(150{ }^{\circ} \mathrm{C}\right)$ favor etching, cation exchange, and alloying, regardless of the precursors used. Under these conditions, the dominant process is determined by the nature of the precursors used. Reactive $S$ precursors (S-ODE/OLAM) combined with unreactive $\mathrm{Zn}$ precursors $\left(\mathrm{ZnSt}_{2}\right)$ result primarily in etching, while the use of reactive $S$ - and $\mathrm{Zn}$-precursors (S-ODE/OLAM and $\mathrm{ZnI}_{2}$ ) results in a combination of etching and $[\mathrm{ZnS}]$ deposition followed by alloying. Unreactive S-precursors (DDT) favor cation exchange involving the $\mathrm{Zn}$-precursors, which is most efficient with more stable $\mathrm{Zn}$-precursors.

High reaction temperatures $\left(210{ }^{\circ} \mathrm{C}\right)$ and less reactive precursors also favor cation exchange followed by alloying. Heteroepitaxial $\mathrm{ZnS}$ shell overgrowth only becomes dominant if reactive $\mathrm{S}$ - and $\mathrm{Zn}$-precursors and high reaction temperatures are used because these conditions shift the balance toward
[ZnS] monomer formation. Nevertheless, a certain degree of $[\mathrm{ZnS}]$ interdiffusion and heterointerfacial alloying still occurs, yielding product NCs, which still show spectral blue-shifts with respect to the seed NCs.

Remarkably, the presence of residual acetate at the surface of the CIS seed NCs is shown to depress etching, cation exchange, and alloying to negligible levels, while facilitating heteroepitaxial shell overgrowth, yielding for the first time CIS/ZnS core/shell NCs displaying red-shifted absorption spectra, in agreement with the spectral shifts expected for a type-I band alignment. This finding highlights the crucial importance of the surface chemistry of the CIS NCs and of the washing procedures used to purify the seed NCs prior to the shelling reaction since residual acetate is only present in samples washed with ethanol. The insights provided by this work pave the way toward the design of improved synthesis strategies to CIS/ZnS core/shell and alloy NCs with tailored elemental distribution profiles, allowing precise tuning of the carrier localization regime and the optoelectronic properties of the resulting materials.

\section{ASSOCIATED CONTENT}

\section{Supporting Information}

The Supporting Information is available free of charge on the ACS Publications website at DOI: 10.1021/acs.chemmater.8b00477.

Representative EDS spectrum of CIS core NCs, XPS spectra, additional HR-HAADF-STEM images of CIS core NCs, alloy and core/shell product NCs, optical spectra of CIS core NCs and product NCs after reaction at different temperatures with different precursors, TEM images of product NCs after reaction with zinc stearate, sulfur, and oleylamine (PDF)

\section{AUTHOR INFORMATION}

\section{Corresponding Author}

*E-mail: c.demello-donega@uu.nl.

ORCID 웅

Ward van der Stam: 0000-0001-8155-5400

Jan P. Hofmann: 0000-0002-5765-1096

Sara Bals: 0000-0002-4249-8017

Celso de Mello Donega: 0000-0002-4403-3627

\section{Present Address}

II W.v.d.S: Optoelectronic Materials Section, Faculty of Applied Sciences, Delft University of Technology, van der Maasweg 9, 2629 HZ Delft, The Netherlands.

\section{Notes}

The authors declare no competing financial interest.

\section{ACKNOWLEDGMENTS}

Annelies van der Bok is gratefully acknowledged for performing the ICP measurements. A.C.B. and C.d.M.D. acknowledge financial support from the division of Chemical Sciences (CW) of The Netherlands Organization for Scientific Research (NWO) under Grant No. ECHO.712.014.001. S.B. and E.B. acknowledge financial support from European Research Council (ERC Starting Grant No. 335078-COLOURATOMS).

\section{REFERENCES}

(1) van der Stam, W.; Berends, A. C.; de Mello Donegá, C. Prospects of Colloidal Copper Chalcogenide Nanocrystals. ChemPhysChem 2016, 17, 559-581. 
(2) Xia, C.; Meeldijk, J. D.; Gerritsen, H. C.; de Mello Donega, C. Highly Luminescent Water-Dispersible NIR-Emitting Wurtzite $\mathrm{CuInS}_{2} / \mathrm{ZnS}$ Core/Shell Colloidal Quantum Dots. Chem. Mater. 2017, 29, 4940-4951.

(3) Kolny-Olesiak, J.; Weller, H. Synthesis and Application of Colloidal $\mathrm{CuInS}_{2}$ Semiconductor Nanocrystals. ACS Appl. Mater. Interfaces 2013, 5, 12221-12237.

(4) Sandroni, M.; Wegner, K. D.; Aldakov, D.; Reiss, P. Prospects of Chalcopyrite-Type Nanocrystals for Energy Applications. ACS Energy Lett. 2017, 2, 1076-1088.

(5) Leach, A. D. P.; Macdonald, J. E. Optoelectronic Properties of $\mathrm{CuInS}_{2}$ Nanocrystals and Their Origin. J. Phys. Chem. Lett. 2016, 7, $572-583$.

(6) Deng, D.; Chen, Y.; Cao, J.; Tian, J.; Qian, Z.; Achilefu, S.; Gu, Y. High-Quality $\mathrm{CuInS}_{2} / \mathrm{ZnS}$ Quantum Dots for In Vitro and In Vivo Bioimaging. Chem. Mater. 2012, 24, 3029-3037.

(7) Wepfer, S.; Frohleiks, J.; Hong, A. R.; Jang, H. S.; Bacher, G.; Nannen, E. Solution-Processed $\mathrm{CuInS}_{2}$-Based White QD-LEDs with Mixed Active Layer Architecture. ACS Appl. Mater. Interfaces 2017, 9, 11224-11230

(8) Meinardi, F.; McDaniel, H.; Carulli, F.; Colombo, A.; Velizhanin, K. A.; Makarov, N. S.; Simonutti, R.; Klimov, V. I.; Brovelli, S. Highly efficient large-area colourless luminescent solar concentrators using heavy-metal-free colloidal quantum dots. Nat. Nanotechnol. 2015, 10, 878-885.

(9) Yang, Y.; Lin, L.; Jing, L.; Yue, X.; Dai, Z. $\mathrm{CuInS}_{2} / \mathrm{ZnS}$ Quantum Dots Conjugating Gd(III) Chelates for Near-Infrared Fluorescence and Magnetic Resonance Bimodal Imaging. ACS Appl. Mater. Interfaces 2017, 9, 23450-23457.

(10) Pan, Z.; Mora-Sero, I.; Shen, Q.; Zhang, H.; Li, Y.; Zhao, K.; Wang, J.; Zhong, X.; Bisquert, J. High-Efficiency "green” quantum Dot Solar Cells. J. Am. Chem. Soc. 2014, 136, 9203-9210.

(11) Li, L.; Pandey, A.; Werder, D. J.; Khanal, B. P.; Pietryga, M.; Klimov, V. I. Efficient Synthesis of Highly Luminescent Copper Indium Sulfide-Based Core/Shell Nanocrystals with Surprisingly Long-Lived Emission. J. Am. Chem. Soc. 2011, 133, 1176-1179.

(12) Berends, A. C.; Rabouw, F. T.; Spoor, F. C. M.; Bladt, E.; Grozema, F. C.; Houtepen, A. J.; Siebbeles, L. D. A.; de Mello Donegá, C. Radiative and Nonradiative Recombination in $\mathrm{CuInS}_{2}$ Nanocrystals and $\mathrm{CuInS}_{2}$-Based Core/Shell Nanocrystals. J. Phys. Chem. Lett. 2016, 7, 3503-3509.

(13) Li, L.; Daou, T. J.; Texier, I.; Kim Chi, T. T.; Liem, N. Q.; Reiss, P. Highly Luminescent $\mathrm{CuInS}_{2} / \mathrm{ZnS}$ Core/Shell Nanocrystals: Cadmium-Free Quantum Dots for In Vivo Imaging. Chem. Mater. 2009, 21, 2422-2429.

(14) De Trizio, L. De; Prato, M.; Genovese, A.; Casu, A.; Povia, M.; Simonutti, R.; Alcocer, M. J. P.; D'Andrea, C.; Tassone, F.; Manna, L. Strongly Fluorescent Quaternary $\mathrm{Cu}-\mathrm{In}-\mathrm{Zn}-\mathrm{S}$ Nanocrystals Prepared from $\mathrm{Cu}_{1-\mathrm{x}} \mathrm{InS}_{2}$ Nanocrystals by Partial Cation Exchange. Chem. Mater. 2012, 24, 2400-2406.

(15) Feng, J.; Sun, M.; Yang, F.; Yang, X. A Facile Approach to Synthesize High-Quality $\mathrm{Zn}(\mathrm{x}) \mathrm{Cu}(\mathrm{y}) \operatorname{InS}(1.5+\mathrm{x}+0.5 \mathrm{y})$ Nanocrystal Emitters. Chem. Commun. 2011, 47, 6422-6424.

(16) Huang, L.; Zhu, X.; Publicover, N. G.; Hunter, K. W.; Ahmadiantehrani, M.; de Bettencourt-Dias, A.; Bell, T. W. Cadmium and Zinc Alloyed Cu-In-S Nanocrystals and Their Optical Properties. J. Nanopart. Res. 2013, 15, 2056.

(17) Uehara, M.; Watanabe, K.; Tajiri, Y.; Nakamura, H.; Maeda, H. Synthesis of $\mathrm{CuInS}_{2}$ Fluorescent Nanocrystals and Enhancement of Fluorescence by Controlling Crystal Defect. J. Chem. Phys. 2008, 129, 134709.

(18) Choi, H. S.; Kim, Y.; Park, J. C.; Oh, M. H.; Jeon, D. Y.; Nam, Y. $\mathrm{S}$. Highly Luminescent, off-Stoichiometric $\mathrm{Cu}_{\mathrm{x}} \mathrm{In}_{\mathrm{y}} \mathrm{S}_{2} / \mathrm{ZnS}$ Quantum Dots for near-Infrared Fluorescence Bio-Imaging. RSC Adv. 2015, 5, 43449-43455.

(19) Park, J.; Kim, S.-W. CuInS 2 /ZnS Core/shell Quantum Dots by Cation Exchange and Their Blue-Shifted Photoluminescence. J. Mater. Chem. 2011, 21, 3745-3750.
(20) Nose, K.; Soma, Y.; Omata, T.; Otsuka-Yao-Matsuo, S. Synthesis of Ternary CuInS 2 Nanocrystals; Phase Determination by Complex Ligand Species. Chem. Mater. 2009, 21, 2607-2613.

(21) Kim, Y.-K.; Ahn, S.-H.; Chung, K.; Cho, Y.-S.; Choi, C.-J. The Photoluminescence of CuInS 2 Nanocrystals: Effect of Non-Stoichiometry and Surface Modification. J. Mater. Chem. 2012, 22, 15161520.

(22) Jana, A.; Lawrence, K. N.; Teunis, M. B.; Mandal, M.; Kumbhar, A.; Sardar, R. Investigating the Control by Quantum Confinement and Surface Ligand Coating of Photocatalytic Efficiency in Chalcopyrite Copper Indium Diselenide Nanocrystals. Chem. Mater. 2016, 28, $1107-1120$.

(23) Komarala, V. K.; Xie, C.; Wang, Y.; Xu, J.; Xiao, M. TimeResolved Photoluminescence Properties of $\mathrm{CuInS}_{2} / \mathrm{ZnS}$ Nanocrystals: Influence of Intrinsic Defects and External Impurities. J. Appl. Phys. 2012, 111, 124314.

(24) Seo, J.; Raut, S.; Abdel-Fattah, M.; Rice, Q.; Tabibi, B.; Rich, R.; Fudala, R.; Gryczynski, I.; Gryczynski, Z.; Kim, W.-J.; et al. TimeResolved and Temperature-Dependent Photoluminescence of Ternary and Quaternary Nanocrystals of $\mathrm{CuInS}_{2}$ with $\mathrm{ZnS}$ Capping and Cation Exchange. J. Appl. Phys. 2013, 114, 94310.

(25) Kim, H.; Han, J. Y.; Kang, D. S.; Kim, S. W.; Jang, D. S.; Suh, M.; Kirakosyan, A.; Jeon, D. Y. Characteristics of $\mathrm{CuInS}_{2} / \mathrm{ZnS}$ Quantum Dots and Its Application on LED. J. Cryst. Growth 2011, 326, 90-93.

(26) Chen, O.; Zhao, J.; Chauhan, V. P.; Cui, J.; Wong, C.; Harris, D. K.; Wei, H.; Han, H.-S.; Fukumura, D.; Jain, R. K.; et al. Compact High-Quality CdSe-CdS Core-Shell Nanocrystals with Narrow Emission Linewidths and Suppressed Blinking. Nat. Mater. 2013, 12, $445-451$.

(27) Smith, A. M.; Lane, L. a.; Nie, S. Mapping the Spatial Distribution of Charge Carriers in Quantum-Confined Heterostructures. Nat. Commun. 2014, 5, 4506.

(28) Kovalenko, M. V.; Manna, L.; Cabot, A.; Hens, Z.; Talapin, D. V.; Kagan, C. R.; Klimov, X. V. I.; Rogach, A. L.; Reiss, P.; Milliron, D. J.; et al. Prospects of Nanoscience with Nanocrystals. ACS Nano 2015, 9, 1012-1057.

(29) Donega, C. de M Synthesis and Properties of Colloidal Heteronanocrystals. Chem. Soc. Rev. 2011, 40, 1512-1546.

(30) Rabouw, F. T.; de Mello Donega, C. Excited-State Dynamics in Colloidal Semiconductor Nanocrystals. Top. Curr. Chem. 2016, 374, 58

(31) Owen, J.; Brus, L. Chemical Synthesis and Luminescence Applications of Colloidal Semiconductor Quantum Dots. J. Am. Chem. Soc. 2017, 139, 10939-10943.

(32) Kim, Y.-K.; Ahn, S.-H.; Choi, G.-C.; Chung, K.-C.; Cho, Y.-S.; Choi, C.-J. Photoluminescence of $\mathrm{CuInS}_{2} /(\mathrm{Cd}, \mathrm{Zn}) \mathrm{S}$ Nanocrystals as a Function of Shell Composition. Trans. Electr. Electron. Mater. 2011, 12, $218-221$.

(33) Zang, H.; Li, H.; Makarov, N. S.; Velizhanin, K. A.; Wu, K.; Park, Y.-S.; Klimov, V. I. Thick-Shell $\mathrm{CuInS}_{2} / \mathrm{ZnS}$ Quantum Dots with Suppressed "Blinking" and Narrow Single-Particle Emission Line Widths. Nano Lett. 2017, 17, 1787-1795.

(34) Park, S. H.; Hong, A.; Kim, J.-H.; Yang, H.; Lee, K.; Jang, H. S. Highly Bright Yellow-Green-Emitting $\mathrm{CuInS}_{2}$ Colloidal Quantum Dots with Core/Shell/Shell Architecture for White Light-Emitting Diodes. ACS Appl. Mater. Interfaces 2015, 7, 6764-6771.

(35) Nam, D.-E.; Song, W.-S.; Yang, H. Facile, Air-Insensitive Solvothermal Synthesis of Emission-Tunable $\mathrm{CuInS}_{2} / \mathrm{ZnS}$ Quantum Dots with High Quantum Yields. J. Mater. Chem. 2011, 21, 1822018226.

(36) Bladt, E.; van Dijk- Moes, R. J. A.; Peters, J.; Montanarella, F.; de Mello Donega, C.; Vanmaekelbergh, D.; Bals, S. Atomic structure of wurtzite CdSe (core)/CdS (giant shell) nanobullets related to epitaxy and growth. J. Am. Chem. Soc. 2016, 138, 14288-14293.

(37) Pietryga, J. M.; Werder, D. J.; Williams, D. J.; Casson, J. L.; Schaller, R. D.; Klimov, V. I.; Hollingsworth, J. A. Utilizing the Lability of Lead Selenide to Produce Heterostructured Nanocrystals with 
Bright, Stable Infrared Emission. J. Am. Chem. Soc. 2008, 130, 48794885 .

(38) Grodzinska, D.; Pietra, F.; van Huis, M. A.; Vanmaekelbergh, D.; de Mello Donega, C. Thermally-Induced Atomic Reconstruction of $\mathrm{PbSe} / \mathrm{CdSe}$ Core/Shell Quantum Dots into $\mathrm{PbSe} / \mathrm{CdSe} \mathrm{Bi}-\mathrm{Hemi}-$ sphere Hetero-Nanocrystals. J. Mater. Chem. 2011, 21, 11556-11565.

(39) Casavola, M.; Van Huis, M. A.; Bals, S.; Lambert, K.; Hens, Z.; Vanmaekelbergh, D. Anisotropic Cation Exchange in $\mathrm{PbSe} / \mathrm{CdSe}$ Core/Shell Nanocrystals of Different Geometry. Chem. Mater. 2012, 24, 294-302.

(40) Standard Thermodynamic Properties of Chemical Substances. In CRC Handbook of Chemistry and Physics; CRC PRESS LLC, 2000. (41) Liebman, J. F.; Holm, T.; Slayden, S. W. The Chemistry of Organomagnesium Compounds; Rappoport, Z., Marek, I., Eds.; John Wiley \& Sons, Ltd: Chichester, UK, 2008; Chapter 2, pp 101-131.

(42) Thomson, J. W.; Nagashima, K.; Macdonald, P. M.; Ozin, G. A. From Sulfur-Amine Solutions to Metal Sulfide Nanocrystals: Peering into the Oleylamine-Sulfur Black Box. J. Am. Chem. Soc. 2011, 133, 5036-5040.

(43) Berends, A. C.; Meeldijk, J. D.; van Huis, M. A.; de Mello Donegá, C. Formation of Colloidal Copper Indium Sulfide Nanosheets by Two-Dimensional Self-Organization. Chem. Mater. 2017, 29, 10551-10560.

(44) Ejder, E. Methods of Representing Emission, Excitation, and Photoconductivity Spectra. J. Opt. Soc. Am. 1969, 59, 223-224.

(45) van der Stam, W.; Berends, A. C.; Rabouw, F. T.; Willhammar, T.; Ke, X.; Meeldijk, J. D.; Bals, S.; de Mello Donega, C. Luminescent $\mathrm{CuInS}_{2}$ Quantum Dots by Partial Cation Exchange in $\mathrm{Cu}_{2-\mathrm{x}} \mathrm{S}$ Nanocrystals. Chem. Mater. 2015, 27, 621-628.

(46) van der Stam, W.; Bladt, E.; Rabouw, F. T.; Bals, S.; de Mello Donega, C. Near-Infrared Emitting CuInSe $2 / \mathrm{CuInS}_{2}$ Dot Core/Rod Shell Heteronanorods by Sequential Cation Exchange. ACS Nano 2015, 9, 11430-11438.

(47) De Trizio, L.; Manna, L. Forging Colloidal Nanostructures via Cation Exchange Reactions. Chem. Rev. 2016, 116, 10852-10887.

(48) Houtepen, A. J.; Hens, Z.; Owen, J. S.; Infante, I. On the Origin of Surface Traps in Colloidal II-VI Semiconductor Nanocrystals. Chem. Mater. 2017, 29, 752-761.

(49) Seah, M. P.; Dench, W. A. Quantitative Electron Spectroscopy of Surfaces: a Standard Data Base for Electron Inelastic Mean Free Paths in Solids. Surf. Interface Anal. 1979, 1, 2-11.

(50) McGuire, G. E.; Schweitzer, G. K.; Carlson, T. A. Study of Core Electron Binding Energies in Some Group IIIa, Vb, and VIb Compounds. Inorg. Chem. 1973, 12, 2450-2453.

(51) Engelhardt, L. M.; Healy, P. C.; Shephard, R. M.; Skelton, B. W.; White, A. H. A Novel Series of 1:1, 2:1 and 3:1 Heterobimetallic Adducts from the Reaction of Copper(I) Halides with Tris(dithiocarbamato)cobalt(III) Complexes. Inorg. Chem. 1988, 27, 2371-2373.

(52) Silvester, E. J.; Grieser, F.; Sexton, B. A.; Healy, T. W. Spectroscopic Studies on Copper Sulfide Sols. Langmuir 1991, 7, 2917-2922.

(53) Siriwardane, R. V.; Poston, J. A.; Fisher, E. P.; Shen, M. S.; Miltz, A. L. Decomposition of the Sulfates of Copper, Iron (II), Iron (III), Nickel, and Zinc: XPS, SEM, DRIFTS, XRD, and TGA Study. Appl. Surf. Sci. 1999, 152, 219-236.

(54) Vollmer, S.; Witte, G.; Wöll, C. Structural Analysis of Saturated Alkanethiolate Monolayers on $\mathrm{Cu}(100)$ : Coexistence of Thiolate and Sulfide Species. Langmuir 2001, 17, 7560-7565.

(55) Lim, H.; Carraro, C.; Maboudian, R.; Pruessner, M. W.; Ghodssi, R. Chemical and Thermal Stability of Alkanethiol and Sulfur Passivated $\operatorname{InP}(100)$. Langmuir 2004, 20, 743-747.

(56) Turo, M. J.; Macdonald, J. E. Crystal-Bound vs Surface-Bound Thiols on Nanocrystals. ACS Nano 2014, 8, 10205-10213.

(57) Gromova, M.; Lefrancois, A.; Vaure, L.; Agnese, F.; Aldakov, D.; Maurice, A.; Djurado, D.; Lebrun, C.; de Geyer, A.; Schulli, T. U.; et al. Growth Mechanism and Surface State of $\mathrm{CuInS}_{2}$ Nanocrystals Synthesized with Dodecanethiol. J. Am. Chem. Soc. 2017, 139, $15748-15759$
(58) Wagener, K.; Batich, C.; Kirsch, B.; Wanigatunga, S. Chain Propagation/Step Propagation Polymerization. III. An XPS Investigation of Poly(oxyethylene)-B-Poly(pivalolactone) Telechelomer. J. Polym. Sci., Part A: Polym. Chem. 1989, 27, 2625-2631.

(59) Yamamoto, F.; Yamakawa, S. Surface Grafting of Polyethylene by Mutual Irradiation in Methyl Acrylate Vapor. III. Quantitative Surface Analysis by X-Ray Photoelectron Spectroscopy. J. Polym. Sci., Polym. Phys. Ed. 1979, 17, 1581-1590.

(60) Vasquez, R. P. CuI by XPS. Surf. Sci. Spectra 1993, 2, 149-154.

(61) Rice, W. D.; Mcdaniel, H.; Klimov, V. I.; Crooker, S. A Magneto-Optical Properties of $\mathrm{CuInS}_{2}$ Nanocrystals. J. Phys. Chem. Lett. 2014, 5, 4105-4109.

(62) Knowles, K. E.; Nelson, H. D.; Kilburn, T. B.; Gamelin, D. R. Singlet-Triplet Splittings in the Luminescent Excited States of Colloidal $\mathrm{Cu}^{+}: \mathrm{CdSe}, \mathrm{Cu}^{+}: \mathrm{InP}$, and $\mathrm{CuInS}_{2}$ Nanocrystals: ChargeTransfer Configurations and Self-Trapped Excitons. J. Am. Chem. Soc. 2015, 137, 13138-13147.

(63) Owen, J. S.; Chan, E. M.; Liu, H.; Alivisatos, A. P. Precursor Conversion Kinetics and the Nucleation of Cadmium Selenide Nanocrystals. J. Am. Chem. Soc. 2010, 132, 18206-18213.

(64) Rempel, J. Y.; Bawendi, M. G.; Jensen, K. F. Insights into the Kinetics of Semiconductor Nanocrystal Nucleation and Growth. J. Am. Chem. Soc. 2009, 131, 4479-4489.

(65) Abe, S.; Čapek, R. K.; de Geyter, B.; Hens, Z. Tuning the Postfocused Size of Colloidal Nanocrystals by the Reaction Rate: From Theory to Application. ACS Nano 2012, 6, 42-53.

(66) Hendricks, M. P.; Campos, M. P.; Cleveland, G. T.; Plante, I. J.; Owen, J. S. A Tunable Library of Substituted Thiourea Precursors to Metal Sulfide Nanocrystals. Science 2015, 348, 1226-1230.

(67) Nakonechnyi, I.; Sluydts, M.; Justo, Y.; Jasieniak, J.; Hens, Z. Mechanistic Insights in Seeded Growth Synthesis of Colloidal Core/ Shell Quantum Dots. Chem. Mater. 2017, 29, 4719-4727.

(68) Groeneveld, E.; Witteman, L.; Lefferts, M.; Ke, X.; Bals, S.; van Tendeloo, G.; de Mello Donega, C. Tailoring ZnSe-CdSe Colloidal Quantum Dots via Cation Exchange: From Core/Shell to Alloy Nanocrystals. ACS Nano 2013, 7, 7913-7930.

(69) Pearson, R. G. Hard and Soft Acids and Bases. Inorg. Chem. 1988, 27, 734-740.

(70) Carbone, L.; Nobile, C.; De Giorgi, M.; Della Sala, F.; Morello, G.; Pompa, P.; Hytch, M.; Snoeck, E.; Fiore, A.; Franchini, I. R.; et al. Synthesis and Micrometer-Scale Assembly of Colloidal CdSe/CdS Nanorods Prepared by a Seeded Growth Approach. Nano Lett. 2007, 7, 2942-2950.

(71) Talapin, D. V.; Koeppe, R.; Götzinger, S.; Kornowski, A.; Lupton, J. M.; Rogach, A. L.; Benson, O.; Feldmann, J.; Weller, H. Highly Emissive Colloidal CdSe/CdS Heterostructures of Mixed Dimensionality. Nano Lett. 2003, 3, 1677-1681.

(72) Kim, D.; Lee, Y. K.; Lee, D.; Kim, W. D.; Bae, W. K.; Lee, D. C. Colloidal Dual-Diameter and Core-Position-Controlled Core/Shell Cadmium Chalcogenide Nanorods. ACS Nano 2017, 11, 1246112472.

(73) Yarema, M.; Xing, Y.; Lechner, R. T.; Ludescher, L.; Dordevic, N.; Lin, W. M. M.; Yarema, O.; Wood, V. Mapping the Atomistic Structure of Graded Core/Shell Colloidal Nanocrystals. Sci. Rep. 2017, 7,11718 .

(74) van der Stam, W.; Geuchies, J. J.; Altantzis, T.; van den Bos, K. H. W.; Meeldijk, J. D.; Van Aert, S.; Bals, S.; Vanmaekelbergh, D.; de Mello Donega, C. Highly Emissive Divalent Ion Doped Colloidal $\mathrm{CsPb}_{1-\mathrm{x}} \mathrm{M}_{\mathrm{x}} \mathrm{Br}_{3}$ Perovskite Nanocrystals through Cation Exchange. J. Am. Chem. Soc. 2017, 139, 4087-4097.

(75) Hughes, J. T.; Navrotsky, A. Enthalpy of Formation of Zinc Acetate Dihydrate. J. Chem. Thermodyn. 2011, 43, 980-982.

(76) Moser, A.; Yarema, M.; Lin, W. M. M.; Yarema, O.; Yazdani, N.; Wood, V. In Situ Monitoring of Cation-Exchange Reaction Shell Growth on Nanocrystals. J. Phys. Chem. C 2017, 121, 24345-24351.

(77) Beberwyck, B. J.; Surendranath, Y.; Alivisatos, A. P. Cation Exchange: A Versatile Tool for Nanomaterials Synthesis. J. Phys. Chem. C 2013, 117, 19759-19770. 
(78) Xia, C.; Winckelmans, N.; Prins, P. T.; Bals, S.; Gerritsen, H. C.; de Mello Donega, C. NIR-Emitting $\mathrm{CuInS}_{2} / \mathrm{ZnS}$ Dot-in-Rod Colloidal Heteronanorods by Seeded Growth. J. Am. Chem. Soc. 2018, DOI: $10.1021 /$ jacs.8b01412. 